S.L. Waters

J.M. Oliver 



\title{
On a poroviscoelastic model for cell crawling
}

\author{
L.S. Kimpton ${ }^{1}$, J.P. Whiteley ${ }^{2}$, S.L. Waters ${ }^{1}$, J.M. Oliver ${ }^{1}$ \\ 1. Mathematical Institute, University of Oxford, \\ 24-29 St. Giles', Oxford, OX1 3LB, UK. \\ 2. Department of Computer Science, University of Oxford, \\ Wolfson Building, Parks Road, Oxford, OX1 3QD, UK.
}

28th March 2013

\begin{abstract}
In this paper a minimal, one-dimensional, two-phase, viscoelastic, reactive, flow model for a crawling cell is presented. Two-phase models are used with a variety of constitutive assumptions in the literature to model cell motility. We use an upperconvected Maxwell model and demonstrate that even the simplest of two-phase, viscoelastic models displays features relevant to cell motility. We also show care must be exercised in choosing parameters for such models as a poor choice can lead to an ill-posed problem. A stability analysis reveals that the initially stationary, spatially uniform strip of cytoplasm starts to crawl in response to a perturbation which breaks the symmetry of the network volume fraction or network stress. We also demonstrate numerically that there is a steady travelling-wave solution in which the crawling velocity has a bell-shaped dependence on adhesion strength, in agreement with biological observation.

Cell motility, Two-phase flow, Viscoelastic, Cell Adhesion
\end{abstract}

\section{Introduction}

\subsection{Biological motivation}

Developing a quantitative understanding of how cells achieve motion will surely revolutionise control of implant colonisation, cancer metastasis and wound healing. Mathematical modelling has made considerable strides towards this goal, but there is still much that remains to be understood (Mogilner, 2009). Before presenting our model and explaining what it adds to the existing literature, we describe briefly the biological basis of cell crawling.

Cells that crawl over flat surfaces such as a microscope slide do so in many ways. Common features include forming flat, sheet-like protrusions, termed lamellipodia, which adhere to the substrate, hauling the bulk of the cell forwards and detaching adhesions at the rear of the cell (Bray, 2001; Mogilner, 2009). It is widely accepted that a cell's actin network is the main generator of protrusive force. Actin monomers polymerize to form filaments that make up part of the cytoskeleton under the close regulation of 
auxiliary proteins that promote polymerization, depolymerization, branching, capping or cross-linking of filaments (Bray, 2001; Pollard and Borisy, 2003; Ridley, 2011; Rottner and Stradal, 2011). Myosin II (referred to simply as 'myosin' henceforth) is a small motor protein important for cell motility, it 'walks' along actin filaments and generates stress in the actin network (Bray, 2001).

Much of the available data pertains to fish epidermal keratocytes, which are popular with experimental scientists, and hence theoreticians, for the simple way in which they crawl. When keratocytes crawl, a large lamellipodium "fans" out ahead of the cell body (Bray, 2001). Their polarization and hence their direction of movement is "persistent," they move quickly with the speed of a "few tens of micrometer per minute" and the cell's shape exhibits "remarkable conservation" (Svitkina et al, 1997). Verkhovsky et al (1999) found nucleus free fragments of keratocytes crawl in much the same way as whole cells. They observed stationary, near symmetric, disc shaped fragments with a ring of high actin density just inside the fragment membrane. A mechanical stimulus triggered polarization of the fragment and transition to a stable locomoting configuration, which has a crescent moon shape, a high actin density at the front of the fragment, and arcs of bundled actin fibres at the rear.

Fluorescent speckle microscopy has provided evidence for strong polymerization of the network at the leading edge and transport of the actin network backwards away from the leading edge, termed retrograde flow (Schaub et al, 2007; Vallotton et al, 2004, 2005). Arc shaped bundles of actin align predominantly parallel to the cell's leading edge where the protrusion joins the main body of the cell (Svitkina et al, 1997). This band of actin is comparable in density to that at the leading edge and exceeds that in the lamellum and the cell body (Svitkina et al, 1997). A forwards flow of the cytosol, the fluid that bathes the actin network, has also been detected in crawling cells (Schaub et al, 2007). Whilst this picture of a steady, translating configuration is broadly true, it neglects some interesting dynamical features of actin networks. Many cells crawl with periodic protrusion and retraction cycles of their leading edge (Burnette et al, 2011). Crawling cells can also exhibit "ruffles", actin-filled folds of the cell membrane which travel backwards over lamella and disappear at the transition to the cell body (Borm et al, 2005; Giannone et al, 2004; Hinz et al, 1999).

Cells adhere by forming attachments between the extra cellular matrix (ECM) and the actin network, via integrins, a class of transmembrane protein, with the help of other auxiliary proteins (Mogilner, 2009). A more detailed description of adhesion complexes can be found in the recent review by Hanein and Horwitz (2012), which estimates that over 200 different molecules have been identified as playing some role in forming adhesion complexes. Experimentalists can vary adhesion strength by changing the substrate a cell crawls over, which makes adhesion a more straightforward parameter to perturb than many other cell properties. As such, there is evidence in the literature that crawling cells exhibit a bell-shaped dependence of speed on adhesion strength: they crawl slower on surfaces that are too sticky or too slippery (Palecek et al, 1997). Burton et al (1999) found that lamellipodia exert a force on the substrate opposing the direction of cell motion. The flanks of the cell exert a large inwards and slightly forwards force on the substrate. The rear of the cell exerts a force on the substrate in the direction of cell motion. A similar experiment in Lee et al (1994) found large, lateral forces directed inwards in the flanks and rear of the cell. However, they did not observe rearward directed force under lamellipodia, 
they found a very small force in the direction of motion at the leading edge of the cell.

\subsection{Mathematical modelling}

The many spatial and temporal scales that exist in motile cells and are important in addressing different questions about cell motility have led to numerous modelling approaches. We shall discuss only the fraction of these most relevant to this paper and refer the interested reader to recent reviews of the field by Mogilner (2009) and Yamaoka et al (2012). Continuum modelling seems to provide a promising framework for modelling cell motility, especially when we wish to model a whole cell. It is typically computationally cheaper than tracking many individual constitutive parts of a cell and allows the modeller to formulate constitutive laws that can be tested against observations at an observable spatial scale. Such models may describe the cell as a viscous fluid (Alt and Tranquillo, 1995; Shao et al, 2012), or a viscoelastic material (Gracheva and Othmer, 2004; Larripa and Mogilner, 2006; Rubinstein et al, 2009; Sarvestani and Jabbari, 2009; Sakamoto et al, 2011; Hodge and Papadopoulos, 2012), typically with additional terms to model the active nature of the cytoskeleton. Both Shao et al (2012) and Gracheva and Othmer (2004) have been able to demonstrate the experimentally observed bell-shaped dependence on adhesion strength. Larripa and Mogilner (2006) present a one-dimensional model which exhibits a travelling wave solution with qualitatively plausible distributions of actin and myosin, but much of the shape of the distribution is prescribed by the boundary conditions. Another interesting continuum model for a crawling cell can be found in Rubinstein et al (2009). It deals only with the cell's lamellipod and they impose a spatially varying adhesion strength in the cell a priori, then solve viscoelastic equations for the actin network in a two-dimensional, realistically shaped domain.

An alternative continuum approach is based on polar gels, see for example Kruse et al (2005); Doubrovinski and Kruse (2007); Joanny et al (2007); Jülicher et al (2007); CallanJones et al (2008); Doubrovinski and Kruse (2008, 2010); Callan-Jones and Jülicher (2011); Doubrovinski and Kruse (2011); Du et al (2012) or Ziebert et al (2012). The cytoplasm is modelled as a fluid with an associated vector field indicating the local actin filament orientation. This modelling draws on techniques from modelling liquid crystals and has delivered a number of interesting results. The filamentous fluid can self-organize into spirals, asters or waves and has solutions that look like polarized travelling cells, see in particular Du et al (2012); Ziebert et al (2012).

This paper is concerned with a related framework for modelling cell motility, namely the two-phase, reactive, flow framework. It describes the cell as made up from a "network' and 'solution' phase. The network phase describes the acto-myosin network with polymerization and depolymerization represented by mass transfer between the phases. It was first considered for modelling cell motility by Dembo et al (1984) and since developed by e.g. Dembo and Harlow (1986); Alt and Dembo (1999); Herant et al (2003); Oliver et al (2005); Rubinstein et al (2005); Zajac et al (2008); Kuusela and Alt (2009); Cogan and Guy (2010) and Kimpton et al (2012). The key advantages of this framework over those discussed above are its ability to: describe polymerization and depolymerization with actin monomers moving out of and into the aqueous cytosol; capture drag between the phases and swelling of the network. It also allows for the description of biomechanical and biochemical interactions and can easily describe the cell membrane 
as a free boundary. Early successes of this approach are discussed further in Kimpton et al (2012) and include the prediction of ruffling dynamics in the actin network (Alt and Dembo, 1999) and the onset of polarization and the existence of travelling wave solutions (Kimpton et al, 2012). The two-phase framework has gained further credence from the work of Moeendarbary et al (2013), who provide substantial experimental evidence that the cell cytoplasm can be understood as a two-phase, poroelastic material. Their results are for much shorter timescales than the crawling timescales we consider and so not at odds with a long-time fluid poroviscoelastic rheology.

In the light of these developments it is increasingly important to understand the two-phase flow framework and the implications of common constitutive assumptions. We will work with the idealised situation of a stripped-down, one-dimensional model of a cell crawling on a flat substrate, since this approach was previously very useful in improving our understanding of the two-phase, reactive, flow network and its applicability to modelling cell motility. In $\S 2$ our analysis is closely related to that in Kimpton et al (2012), who dealt with the purely viscous regime.

\subsection{Paper outline}

In this paper we first formulate the viscoelastic model in $\S 2$. A potential hodograph transform enables us to identify contraction of the actin network as a destabilising mechanism and to see explicitly the history dependence introduced by a viscoelastic rheology. Results of a stability analysis presented in $\S 3$ reveal that a perturbation must break the symmetry of the uniform stationary state in order to cause the strip to crawl. The stability analysis also shows that in some parameter regimes the growth rate plateaus for large wavenumbers, meaning that the solution of the initial value problem is sensitive to both the modes and the relative amplitude of the modes contained in the initial conditions. It reveals regions in parameter space for which the model becomes ill-posed. We show in $\S 4$ that in a small growth rate limit of the model, certain types of initial condition allow us to predict in which direction the strip will start to crawl. In $\S 5$ we solve the viscoelastic model numerically and investigate the effect of Deborah number and strength of cell-substrate adhesion. We demonstrate that the model exhibits a steady travellingwave solution, the velocity of which has a bell-shaped dependence on adhesion strength. Finally the results of the paper and directions for future work are discussed in $\S 6$.

\section{Model formulation}

Characterizing cell rheology is particularly difficult because cells respond to mechanical tests altering the very properties experimentalists are attempting to measure (Mofrad, 2009). Experimental evidence suggesting that the actomyosin network in cells is shorttime (seconds) elastic and long-time viscous has been discussed in the recent review by Levayer and Lecuit (2011). We focus on the simplest possible viscoelastic constitutive law with this property. In particular, we will restrict our model to have only one viscoelastic relaxation timescale and employ an upper-convected Maxwell model to describe the rheology of the actin network. These modelling assumptions are supported by Jülicher et al (2007) who use a convected Maxwell model to describe the actin network, although their 
governing equations are more complicated because the rheology is coupled to a polarization field for the actin fibres. The lamellipod is described as an upper-convected Maxwell fluid in Rubinstein et al (2009). Both these models are single phase models. For further justification we appeal to models developed in Knapp et al (1997) and Ohsumi et al (2008) for a similar system. They describe a hydrated collagen network as a two-phase fluid, where the collagen network is an upper-convected Maxwell fluid. In Knapp et al (1997) the model predictions are compared to measurements from a simple experimental set-up and found to be in good agreement.

\subsection{Mass and momentum conservation}

We follow Alt and Dembo (1999) and Kimpton et al (2012) and use the reactive, twophase flow framework in one dimension to model a crawling cell. The distance along a strip of cytoplasm parallel to the direction of motion is denoted by $x$. Continuity of the network and solution phases is given by

$$
\frac{\partial \theta}{\partial t}+\frac{\partial}{\partial x}(\theta u)=-J, \quad \frac{\partial}{\partial t}(1-\theta)+\frac{\partial}{\partial x}((1-\theta) v)=J
$$

where $t$ denotes times, $\theta(x, t)$ is the network volume fraction, $u(x, t)$ is the network velocity and $v(x, t)$ is the solution velocity. Polymerization $(J<0)$ and depolymerization $(J>0)$ of the actin network are modelled via the mass transfer term $J$. The force balance on the network is given by

$$
-\theta \frac{\partial p}{\partial x}-\frac{\partial \Psi}{\partial x}+\frac{\partial}{\partial x}(\theta \sigma)=H(u-v)+\beta u
$$

where $p$ is the hydrodynamic pressure, $\Psi$ describes the swelling or contractile pressure in the network, $\sigma$ is the network stress, $H$ is the hydrodynamic drag between network and solution phases and $\beta$ describes the cell's adhesion to the substrate it crawls over. This equation introduces two new dependent variables $p$ and $\sigma$, while the functions $\Psi, H$ and $\beta$ are prescribed constitutively. The swelling and contractile pressure can be thought of as a modification to the local pressure that drives the flow of the network. We assume that the network swells or contracts depending on the local volume fraction of network. When $\Psi^{\prime}(\theta)>0$ the $\Psi$ term in (2) acts to drive flow of the network down its volume fraction gradient modelling network swelling, when $\Psi^{\prime}(\theta)<0$ the $\Psi$ term in (2) acts to drive flow of the network up its volume fraction gradient modelling network contraction. Following Ohsumi et al (2008), the upper-convected Maxwell constitutive equation for the network stress is taken to be given by

$$
\frac{1}{2 G}\left(\frac{\partial \sigma}{\partial t}+u \frac{\partial \sigma}{\partial x}-2 \sigma \frac{\partial u}{\partial x}\right)+\frac{\sigma}{2 \mu_{n}}=\frac{\partial u}{\partial x}+\left(\frac{\nu}{1-2 \nu}\right) \frac{\partial u}{\partial x},
$$

where $G$ is the shear modulus, $\mu_{n}$ is the shear viscosity and $\nu$ is the Poisson ratio of the network. The viscosity of the network, the hydrodynamic drag and the adhesion between the cell and the substrate resist and balance the pressure driven flow of the network.

The force balance on the solution phase is given by

$$
-(1-\theta) \frac{\partial p}{\partial x}=H(v-u)
$$


representing our assumption of Darcy flow of the solution through the porous medium formed by the actin network. Summing (2) and (4) we find that the force balance on the two-phase mixture is given by

$$
\frac{\partial \Sigma}{\partial x}=\beta u,
$$

where $\Sigma$, the total mixture stress, is given by

$$
\Sigma=-p-\Psi+\theta \sigma .
$$

To close the system we require constitutive laws for $J, \Psi, H$ and $\beta$. We shall keep the calculations that follow as general as possible, by assuming only that $J, \Psi, H$ and $\beta$ are functions of $\theta$. Where it is necessary to specify the form of these functions, for example in numerical simulations, we shall use the following constitutive laws

$$
\begin{gathered}
J(\theta)=\frac{\theta-\theta_{E}}{T}, \quad \Psi^{\prime}(\theta)=\Psi_{0} \frac{\left(\theta-\theta_{L}\right)\left(\theta-\theta_{R}\right)}{(1-\theta)}, \\
H(\theta)=H_{0} \theta(1-\theta), \quad \beta(\theta) \stackrel{=}{=} \beta_{0} \theta .
\end{gathered}
$$

The assumption behind the constitutive law for $J$ is that the polymerization/depolymerization kinetics are linear and drive the local network volume fraction towards some equilibrium volume fraction $\theta_{E}$ on a timescale $T$. In prescribing the swelling/contractile pressure we assume that the network contracts at intermediate volume fractions $\theta_{L}<\theta<\theta_{R}$ where there is sufficient network for myosin to bind and cause contraction. We assume that at high network volume fractions, there is more cross-linking between actin fibres making the network harder to contract and at low network volume fraction there is insufficient myosin the cause contraction. We select $\theta_{L}<\theta_{E}<\theta_{R}$, so that the polymerization/depolymerization kinetics drive the network volume fraction into the contractile regime. A more detailed discussion of the constitutive assumption for the swelling/contractile pressure can be found in Kimpton et al (2012). The remaining constitutive laws for $H$ and $\beta$ are the simplest biologically plausible assumptions that we can make. Finally, further following our 'simplest problem first' philosophy, we assume throughout this paper that $G, \mu_{n}$ and $\nu$ are constant, although in reality they could depend on a number of things including the network volume fraction $\theta$, the network stress, cross-linking molecules in the network or biochemical signalling molecules in the cell.

\subsection{Boundary and initial conditions}

In order to close the viscoelastic model described in $\S 2.1$ we must impose boundary and initial conditions. We seek to solve the governing equations (1), (2), (3) and (4) on the strip $a(t) \leqslant x \leqslant b(t)$, which is aligned with the direction of cell motion, so that the strip moves and $a(t)$ and $b(t)$ must be determined as part of the solution. We impose no flux of network or solution and no mixture stress at the ends of the strip:

$$
\begin{array}{lll}
\text { at } x=a(t), & u=\dot{a}, & v=\dot{a}, \quad \Sigma=0, \\
\text { at } x=b(t), & u=\dot{b}, \quad v=\dot{b}, \quad \Sigma=0 .
\end{array}
$$

The system also requires initial conditions

$$
a(0)=0, \quad b(0)=L ; \quad \text { for } 0<x<L, \quad \theta(x, 0)=\theta^{*}(x), \quad \sigma(x, 0)=\sigma^{*}(x) ;
$$

where $L$ is the initial length of the domain and $\theta^{*}$ and $\sigma^{*}$ are the initial network volume fraction and stress, respectively. 


\subsection{Reduced system}

We introduce the mixture velocity defined

$$
V=\theta u+(1-\theta) v
$$

and see from summing $(1 a)$ and $(1 b)$ that $V$ depends only on time. We can use the mixture velocity to eliminate the pressure $p$ and the solution velocity $v$, to obtain the governing equations

$$
\frac{\partial \theta}{\partial t}+\frac{\partial}{\partial x}(\theta u)=-J, \quad-\frac{\partial \Psi}{\partial x}+\frac{\partial}{\partial x}(\theta \sigma)=\alpha(u-V)+\beta u,
$$

and (3), where

$$
\alpha(\theta)=H(\theta) /(1-\theta)^{2} .
$$

These governing equations must be solved subject to

$$
u(a(t), t)=V(t), \quad u(b(t), t)=V(t),
$$

where $a(t)$ is given by

$$
a(t)=\int_{0}^{t} V(\tau) \mathrm{d} \tau
$$

and $b(t)=a(t)+L$. Finally we obtain a solvability condition for $V(t)$ by integrating the global force balance (5) and applying the no stress boundary conditions $(9 c)$ and $(10 c)$ to find

$$
\int_{a(t)}^{b(t)} \beta(\theta(x, t)) u(x, t) \mathrm{d} x=0 .
$$

\subsection{Nondimensionalization}

We nondimensionalize the variables as follows

$$
x=L x^{\dagger}, \quad t=T t^{\dagger}, \quad u=\frac{L}{T} u^{\dagger}, \quad V=\frac{L}{T} V^{\dagger}, \quad \sigma=\frac{2 \mu_{1}}{T} \sigma^{\dagger}, \quad a=L a^{\dagger}, \quad b=L b^{\dagger},
$$

where $L$ is the initial length of the strip and $T$ is the timescale for network turnover. Noting the form of the viscous term in (3) the scaling for the network viscosity is chosen so that

$$
\mu_{n}=\mu_{1}\left(\frac{1-2 \nu}{1-\nu}\right)
$$

The magnitude of the network viscosity, $\mu_{n}$, is contained in $\mu_{1}$, with a correction for the network Poisson ratio. We rescale the constitutive equations by setting

$$
J=\frac{1}{T} J^{\dagger}, \quad \Psi=\frac{2 \mu_{1}}{T} \Psi^{\dagger}, \quad \alpha=\frac{2 \mu_{1}}{L^{2}} \alpha^{\dagger}, \quad \beta=\frac{2 \mu_{1}}{L^{2}} \beta^{\dagger},
$$

where a dagger denotes a dimensionless quantity. 


\subsection{Travelling frame}

We move to a frame that travels with the strip, at speed $V(t)$, according to the transformation

$$
\begin{array}{cc}
x^{\dagger}=\int_{0}^{t^{\dagger}} V^{\dagger}(\tau) \mathrm{d} \tau+\hat{x}, & u^{\dagger}\left(x^{\dagger}, t^{\dagger}\right)=V^{\dagger}\left(t^{\dagger}\right)+\hat{u}\left(\hat{x}, t^{\dagger}\right), \\
\theta^{\dagger}\left(x^{\dagger}, t^{\dagger}\right)=\hat{\theta}\left(\hat{x}, t^{\dagger}\right), & \sigma^{\dagger}\left(x^{\dagger}, t^{\dagger}\right)=\hat{\sigma}\left(\hat{x}, t^{\dagger}\right),
\end{array}
$$

of the nondimensional versions of (3), (11)-(13) and (15)-(17). After dropping hats and daggers, the governing equations to be solved on $0 \leqslant x \leqslant 1$ for $t>0$ are given by

$$
\begin{aligned}
\frac{\partial \theta}{\partial t}+\frac{\partial}{\partial x}(\theta u) & =-J \\
-\frac{\partial \Psi}{\partial x}+\frac{\partial}{\partial x}(\theta \sigma) & =\alpha u+\beta(u+V), \\
D\left(\frac{\partial \sigma}{\partial t}+u \frac{\partial \sigma}{\partial x}-2 \sigma \frac{\partial u}{\partial x}\right)+\sigma & =\frac{\partial u}{\partial x}
\end{aligned}
$$

where $D=\mu_{1}(1-2 \nu) /(G T(1-\nu))$, the dimensionless ratio of the network's elastic relaxation timescale to the network turnover timescale. These are subject to the boundary and initial conditions

$$
\begin{aligned}
& \text { for } t>0, \quad u(0, t)=0, \quad u(1, t)=0 ; \\
& \text { for } 0 \leqslant x \leqslant 1, \quad \theta(x, 0)=\theta^{*}(x), \quad \sigma(x, 0)=\sigma^{*}(x),
\end{aligned}
$$

and the solvability condition

$$
\langle\beta(u+V)\rangle=0
$$

where

$$
\langle f\rangle=\int_{0}^{1} f(x) \mathrm{d} x
$$

So we see that

$$
V=-\langle\beta u\rangle /\langle\beta\rangle
$$

and the model displays the biologically observed retrograde flow on the scale of the strip. The travelling strip moves forwards as the network flows backwards. The nondimensional constitutive laws for $J, \Psi, \alpha$ and $\beta$ are given by

$$
J(\theta)=\theta-\theta_{E}, \quad \Psi^{\prime}(\theta)=\Psi^{*} \frac{\left(\theta-\theta_{L}\right)\left(\theta-\theta_{R}\right)}{(1-\theta)},
$$

together with

$$
\alpha(\theta)=\alpha^{*} \theta /(1-\theta), \quad \beta(\theta)=\beta^{*} \theta .
$$

The dimensionless parameters in (31)-(32) are given by

$$
\Psi^{*}=\frac{\Psi_{0} T}{2 \mu_{1}}, \quad \alpha^{*}=\frac{H_{0} L^{2}}{2 \mu_{1}}, \quad \beta^{*}=\frac{\beta_{0} L^{2}}{2 \mu_{1}},
$$

representing the relative strengths of the swelling/contractile stress, the hydrodynamic drag and the cell-substrate adhesion to the network viscosity. The model has four additional dimensionless parameters, namely the Deborah number $D$ and the network parameters $\theta_{L}, \theta_{E}$ and $\theta_{R}$. 


\subsection{Dimensionless parameters}

The Deborah number $D$ is the nondimensional ratio of the relaxation timescale of the network to the observation timescale (Howell et al, 2009), where we choose to observe the crawling cell on the timescale $T$. This is reasonable because $T$ is the order of minutes and cells crawl at speeds on the order of microns to tens of microns per minute. When $D$ is small the network behaves as a viscous fluid and when $D$ is large the network behaves as an elastic solid. Estimates for the viscoelastic relaxation timescale of the actin network of at most a few seconds can be found in Bausch et al (1998); Kole et al (2005); Wottawah et al (2005). If we take as an upper bound $\mu_{n} / G=6$ seconds, this leads to a value for our Deborah number of $D=0.1$. We note that if we set $D=0$ we recover the minimal model presented in Kimpton et al (2012) where the network is modelled as a very viscous fluid with an effective viscosity. We note that in our one-dimensional framework, the shear and bulk contributions to the viscosity cannot be distinguished, as shown by (19). Therefore, in the absence of reliable estimates for the Poisson ratio of the actin network, $\nu$, and noting the large range of experimental values for the network viscosity discussed in Kimpton et al (2012), we consider reasonable values for $\mu_{1}$ in (32) to be in the range $10^{-4}-1 \mathrm{kPa}$ min. Suitable ranges of values for the other dimensionless parameters can also be taken from Kimpton et al (2012): $\Psi^{*}=0-5 \times 10^{6}, \alpha^{*}=4 \times 10^{-5}-4 \times 10^{3}$, $\beta^{*}=2-2 \times 10^{7}, \theta_{L}=0.01, \theta_{E}=0.02$ and $\theta_{R}=0.03$.

\subsection{Reduction to single equation for $J=0$}

In order to improve our understanding of the nature of the governing equations we consider a potential hodograph transform by introducing the integrated mass variable

$$
s=\int_{0}^{\chi(s, t)} \theta(\xi, t) \mathrm{d} \xi, \quad \chi(0, t)=0,
$$

and transforming the governing equations. The details can be found in appendix A, but in the case where there is no polymerization or depolymerization $(J=0)$ the governing equations can be reduced to

$$
\frac{\partial \phi}{\partial t}=\frac{\partial}{\partial s}\left(A(\phi) \frac{\partial \phi}{\partial s}+B(\phi) \frac{\partial}{\partial s}\left(\phi e^{-t / D}\left(\frac{\Lambda^{*}}{\phi^{* 2}}+\frac{1}{D} \int_{0}^{t} \frac{e^{\xi / D}}{\phi^{3}} \frac{\partial \phi}{\partial \xi} \mathrm{d} \xi\right)\right)-C(\phi) V\right)
$$

an integro-partial-differential equation for $\phi=1 / \theta$, with one extra unknown, $V(t)$, which is determined by a transformed version of the integral constraint (28). The coefficients are given by

$$
\begin{aligned}
A(\phi) & =\frac{\Psi^{\prime}(1 / \phi)}{\phi^{3}(\alpha(1 / \phi)+\beta(1 / \phi))}, \\
B(\phi) & =\frac{1}{\phi(\alpha(1 / \phi)+\beta(1 / \phi))}, \\
C(\phi) & =\frac{\beta(1 / \phi)}{(\alpha(1 / \phi)+\beta(1 / \phi))},
\end{aligned}
$$

and $\phi^{*}$ and $\Lambda^{*}$ are the initial conditions for $\phi$ and the transformed network stress respectively. When the network volume fraction is in the contractile regime $A<0$, conversely 
$A>0$ when the network is in the swelling regime. Therefore, the potential hodograph transform is informative in identifying that contraction of the network can be viewed as a destabilising, backwards diffusion term which drives the cell motion. We see explicitly that the viscoelastic network rheology introduces a term which is nonlocal in time. We also see that the timescale over which the network's flow history effects the current network configuration decreases as $D \rightarrow 0$. We proceed to consider a stability analysis of the model.

\section{$3 \quad$ Linear stability analysis}

In this section we investigate the stability and posedness of the governing equations. We analyse how the strip responds to a small perturbation of the network volume fraction and network stress about their respective equilibrium values, to gain insight into the polarization and determination of crawling direction in cells.

\subsection{Unbounded domain}

On the domain $-\infty<x<\infty$ we note that the spatially uniform steady state $u=u_{E}$, $V=V_{E}, \theta=\theta_{E}, \sigma=0$, where $u_{E}, V_{E}$ and $\theta_{E}$ are constants, satisfies (23)-(25) if and only if $J\left(\theta_{E}\right)=0$ and $\alpha_{E} u_{E}+\beta_{E}\left(u_{E}+V_{E}\right)=0$. A subscript $E$ on a constitutive relation indicates that the function is evaluated at the equilibrium network volume fraction (thus, $\alpha_{E}=\alpha\left(\theta_{E}\right)$ etc). The problem is dramatically simplified by setting $u_{E}=V_{E}=0$ and since, when we introduce the boundary conditions in $\S 3.2$ the stationary steady state is the selected one, we proceed to analyse this case. The aim is to understand how small perturbations to the stationary steady state behave.

We consider a small, order $\varepsilon$, perturbation to the steady state by seeking a solution to $(23)-(25)$ of the form

$$
u \sim \varepsilon u_{1}+\ldots, \quad V \sim \varepsilon V_{1}+\ldots, \quad \theta \sim \theta_{E}+\varepsilon \theta_{1}+\ldots, \quad \sigma \sim \varepsilon \sigma_{1}+\ldots
$$

At $O(\varepsilon)$ we obtain the following linearized system of equations:

$$
\begin{aligned}
\frac{\partial \theta_{1}}{\partial t}+\theta_{E} \frac{\partial u_{1}}{\partial x} & =-\theta_{1} \\
-\Psi_{E}^{\prime} \frac{\partial \theta_{1}}{\partial x}+\theta_{E} \frac{\partial \sigma_{1}}{\partial x} & =\alpha_{E} u_{1}+\beta_{E}\left(u_{1}+V_{1}\right), \\
D \frac{\partial \sigma_{1}}{\partial t}+\sigma_{1} & =\frac{\partial u_{1}}{\partial x}
\end{aligned}
$$

where a prime' on a function denotes differentiation of that function with respect to the argument $\theta$ (thus $\Psi_{E}^{\prime}=\partial \Psi / \partial \theta\left(\theta_{E}\right)$ etc). We have used the fact that our scalings in $\S 2.4$ imply $J_{E}^{\prime}=1$. Now we can eliminate $u_{1}$ and $\sigma_{1}$ to obtain the third-order partial differential equation

$$
\left[D \frac{\partial}{\partial t}+1\right]\left(\frac{\alpha_{E}+\beta_{E}}{\theta_{E}}\left(\theta_{1}+\frac{\partial \theta_{1}}{\partial t}\right)-\Psi_{E}^{\prime} \frac{\partial^{2} \theta_{1}}{\partial x^{2}}\right)=\frac{\partial^{2}}{\partial x^{2}}\left(\theta_{1}+\frac{\partial \theta_{1}}{\partial t}\right) .
$$


If we consider $\theta_{1}$ to be proportional to $\exp (i k x+\Omega(k) t)$ for real $k$, then a perturbation with wavenumber $k$ grows at the rate $R e[\Omega(k)]$ where $\Omega$ is given by

$$
\Omega^{2}+b \Omega+c=0
$$

with coefficients given by

$$
b(k)=1+\frac{1}{D}+\frac{\theta_{E}}{\alpha_{E}+\beta_{E}}\left[\Psi_{E}^{\prime}+\frac{1}{D}\right] k^{2}, \quad c(k)=\frac{1}{D}+\frac{\theta_{E}\left(\Psi_{E}^{\prime}+1\right)}{D\left(\alpha_{E}+\beta_{E}\right)} k^{2},
$$

for $D \neq 0$ and $\alpha_{E}+\beta_{E} \neq 0$.

The largest growth rate of a perturbation with wavenumber $k$ is given by the real part of $\Omega(k)_{+}$, where

$$
\Omega_{ \pm}(k)=\frac{-b \pm \sqrt{b^{2}-4 c}}{2} .
$$

Manipulating the discriminant, $\Delta=b^{2}-4 c$, we find that

$$
\Delta=\left(\frac{1}{D}-1\right)^{2}+\frac{2 \theta_{E}}{\alpha_{E}+\beta_{E}}\left(\frac{1}{D}-1\right)\left(\frac{1}{D}-\Psi_{E}^{\prime}\right) k^{2}+\left(\frac{\theta_{E}}{\alpha_{E}+\beta_{E}}\right)^{2}\left(\frac{1}{D}+\Psi_{E}^{\prime}\right)^{2} k^{4} .
$$

Since the equilibrium volume fraction is chosen so that the kinetics drive the network volume fraction into the contractile regime, $\Psi_{E}^{\prime}<0$. If $D<1$ the discriminant is positive and there exist two distinct real roots for all wavenumbers. If alternatively, $D>1$ there will be an interval of wavenumbers for which the discriminant is negative, but the discriminant will be positive for sufficiently large $k$. Where $\Delta(k)<0$ the growth rate of the perturbation is given by $\operatorname{Re}[\Omega(k)]=-b / 2$. We have identified the size of $D$ as important in classifying the growth rate, but we postpone a discussion of the implications in terms of relaxation and network turnover timescales until the end of this section.

In order to construct the shape of the growth rate curves we first note that a flat perturbation $(k=0)$ is stable. To further analyse the growth rates we introduce the notation $b=b_{1}+b_{2} k^{2}, c=c_{1}+c_{2} k^{2}$ and look for extrema by setting $d \Omega / d k^{2}=0$, which implies

$$
b_{2} \sqrt{\Delta}= \pm\left(b_{1} b_{2}+b_{2}^{2} k^{2}-2 c_{2}\right) .
$$

Since this can't be satisfied if $\Delta<0$ (except trivially by $b_{2}=c_{2}=0$ ) we proceed to consider $\Delta>0$ and square both sides, rearranging to obtain

$$
b_{1} b_{2} c_{2}-b_{2}^{2} c_{1}-c_{2}^{2}=0
$$

which has no $k$ dependence, implying that the growth rate curves have no extrema. Therefore, if $D<1$ then the growth rate curves are monotonic. However, if $D>1$ there is an interval of wavenumbers for which $\Delta<0$ and the real part of the growth rate may have discontinuous derivatives at either end of the interval. Now we turn our attention to the growth rate curves at large $k$. Expanding (46) for large $k$ we see that if $b_{2}>0$ then $\Omega_{+} \rightarrow-c_{2} / b_{2}$ and $\Omega_{-} \sim-b_{2} k^{2}$ as $k \rightarrow \infty$. Alternatively, if $b_{2}<0$ then $\Omega_{+} \sim-b_{2} k^{2}$ and $\Omega_{-} \rightarrow-c_{2} / b_{2}$ as $k \rightarrow \infty$.

We are now in a position to characterize the growth rates in terms of the parameter values. We consider $D<1$ where the elastic relaxation timescale is smaller than the network turnover timescale and note that this implies $\Delta>0$. The growth rate is monotonic 
and there exist three regimes based on the size of $\Psi_{E}^{\prime}$. Firstly, if $\Psi_{E}^{\prime}<-1 / D$ then $\Omega_{+}$ is positive and unbounded for large $k$, so the linearized system is ill-posed. Secondly, if $-1 / D<\Psi_{E}^{\prime}<-1$ then $\Omega_{+}$is initially negative and grows monotonically to plateau at $\Omega_{\infty}$ where

$$
\Omega_{\infty}=-\frac{c_{2}}{b_{2}}=-\frac{1+\Psi_{E}^{\prime}}{1+D \Psi_{E}^{\prime}}
$$

and $\Omega_{\infty}>0$. This is the interesting case, where all wavenumbers greater than some critical wavenumber, $k_{c}$ are unstable but grow at a bounded rate. We see from $(45 b)$ that

$$
k_{c}^{2}=-\frac{\alpha_{E}+\beta_{E}}{\theta_{E}\left(1+\Psi_{E}^{\prime}\right)} .
$$

Thirdly, when $\Psi_{E}^{\prime}>-1, \Omega_{+}$is initially negative and grows monotonically to $\Omega_{\infty}$ which is negative, so all wavenumbers are stable. Figure 1 depicts the growth rate, $\Omega_{+}$, and the smaller root, $\Omega_{-}$, in all three cases.

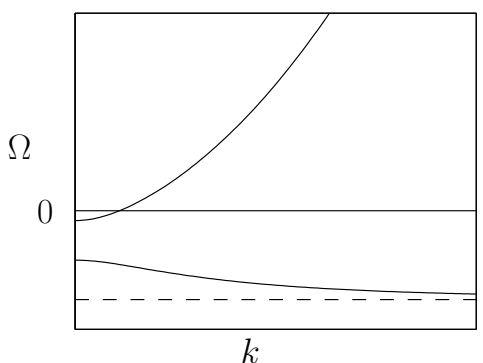

(a) $\Psi_{E}^{\prime}<-1 / D$

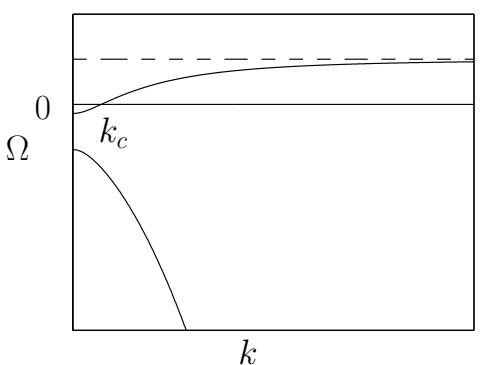

(b) $-1 / D<\Psi_{E}^{\prime}<-1$

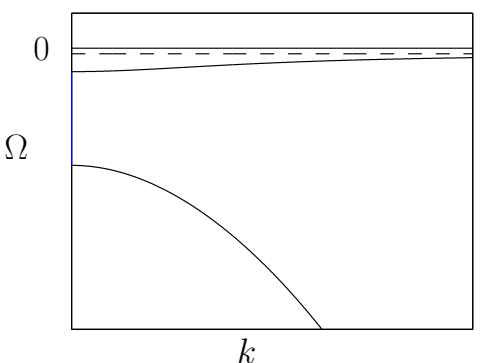

(c) $-1<\Psi_{E}^{\prime}$

Figure 1: The roots of (46) are plotted as a function of $k$, for $D<1$, for three cases distinguished by the size of $\Psi_{E}^{\prime}$. In all three cases $\Omega_{+}(0)=-1, \Omega_{-}(0)=-1 / D$ and $\Omega_{\infty}$, given by (47), is marked as a dashed line.

If $D>1$, so that the elastic relaxation timescale is larger than the network turnover timescale, we again work through the three cases for $\Psi_{E}^{\prime}$. We find that in both the cases where $\Psi_{E}^{\prime}<-1$ and $-1<\Psi_{E}^{\prime}<-1 / D, \Omega_{+}$, is proportional to $k^{2}$ for large wavenumbers and the problem is ill-posed. In the final case where $-1 / D<\Psi_{E}^{\prime}, \Omega_{\infty}<0$ and all wavenumbers are stable. All three of these situations are shown in Figure 2, along with the corresponding smaller root of (46).

In the borderline case where $D=1$, the growth rate equation (44) can be factorized to reveal the roots

$$
\Omega=-1, \quad \Omega=-1-\frac{\theta_{E}\left(1+\Psi_{E}^{\prime}\right)}{\alpha_{E}+\beta_{E}} k^{2} .
$$

If $\Psi_{E}^{\prime}>-1$ then the steady state is stable to perturbations for all wavenumbers $k$. If $\Psi_{E}^{\prime}<-1$ then the largest growth rate grows proportionally to $k^{2}$ and the system is ill-posed.

In summary, for the problem to not be ill-posed we require $\Psi_{E}^{\prime}>-1 / D$, then for the growth rate to plateau to a positive value we require $\Psi_{E}^{\prime}<-1$. It is only possible to satisfy both of these criteria when $D<1$, i.e. the elastic relaxation timescale is smaller than 


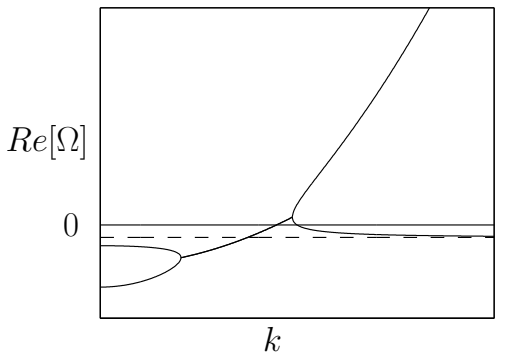

(a) $\Psi_{E}^{\prime}<-1$

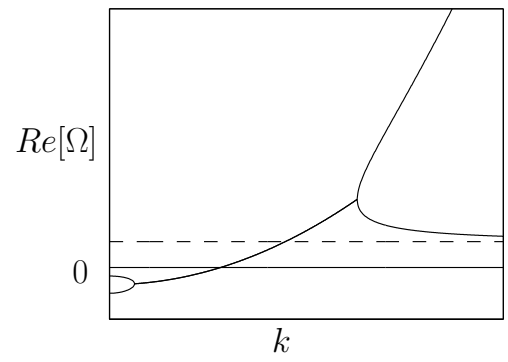

(b) $-1<\Psi_{E}^{\prime}<-1 / D$

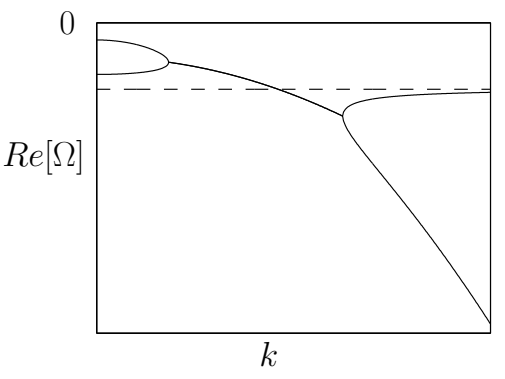

(c) $-1 / D<\Psi_{E}^{\prime}$

Figure 2: The real part of the roots of (46) are plotted as a function of $k$, for $D>1$, for three cases distinguished by the size of $\Psi_{E}^{\prime}$. In all three cases $\Omega_{+}(0)=-1 / D, \Omega_{-}(0)=-1$ and $\Omega_{\infty}$, given by (47), is marked as a dashed line.

the network turnover time. This is a reasonable constraint on the parameter values for our model. If the elastic relaxation time was long in comparison to the network turnover time, it would suggest a different, long-time elastic rheology for the actin network. This would require a different type of viscoelastic model. So one result of the stability analysis is to demonstrate that in order for the model to not be ill-posed we must pick parameters consistent with the short-time-elastic assumption that underpins our upper-convected Maxwell constitutive law for the network stress. We note that the plots in Figure 2 for $D>1$ display an interval in $k$ where the roots of (46) are complex conjugate pairs. If we consider just the parameter sub-regime $\Psi_{E}^{\prime}>-1 / D$ that leads to a well-posed model (see Figure 2(c)), the stability analysis predicts that for a range of wavenumbers, perturbations to the stationary steady state will travel as they decay in amplitude. This could represent actin ruffles that travel over lamellipodia.

\subsection{Effect of boundary conditions}

Now we restrict our stability analysis to the problem domain $0 \leqslant x \leqslant 1$ and ensure our perturbations satisfy the boundary conditions $u(0, t)=u(1, t)=0$. We seek solutions to (40)-(42) of the form $\theta_{1}=\hat{\theta}_{1}(x) e^{\omega t}, u_{1}=\hat{u}_{1}(x) e^{\omega t}, \sigma_{1}=\hat{\sigma}_{1}(x) e^{\omega t}$ and $V=\hat{V}_{1} e^{\omega t}$, such that $\hat{u}_{1}(0)=\hat{u}_{1}(1)=0$. After dropping hats and eliminating $\theta_{1}$ and $\sigma_{1}$, we obtain the second-order, constant-coefficient, linear, differential equation for $u_{1}$ forced by $V_{1}$, viz.

$$
\left[\frac{\theta_{E} \Psi_{E}^{\prime}}{\omega+1}+\frac{\theta_{E}}{D \omega+1}\right] u_{1}^{\prime \prime}-\left(\alpha_{E}+\beta_{E}\right) u_{1}=\beta_{E} V_{1}
$$

for $\omega+1 \neq 0$ and $D \omega+1 \neq 0$. (We note from the previous section that for general parameters, $\omega=-1$ and $D \omega=-1$ only at $k=0$.) This must be solved subject to the linearized version of (28) given by

$$
\left\langle\beta_{E}\left(u_{1}+V_{1}\right)\right\rangle=0 .
$$

For $V_{1}=0$ we find the following eigensolutions

$$
u_{1}=\sin (n \pi x) e^{\omega_{n} t}, \quad \theta_{1}=-\frac{\theta_{E} n \pi}{\omega_{n}+1} \cos (n \pi x) e^{\omega_{n} t}, \quad \sigma_{1}=\frac{n \pi}{D \omega_{n}+1} \cos (n \pi x) e^{\omega_{n} t},
$$


where the real part is understood for the rest of this paper, $\omega_{n}=\Omega_{+}(n \pi)$ and $(51)$ requires $n$ to be an even integer. So we see that small perturbations to the network volume fraction or network stress in the spatially uniform, stationary strip which are symmetric about $x=1 / 2$ do not cause the strip to move. We exploit the symmetry in the boundary conditions for $u_{1}$ to find solutions to the inhomogeneous differential equation where $V_{1} \neq 0$ as follows

$$
\begin{aligned}
u_{1} & =\frac{\beta_{E} V_{1}}{\alpha_{E}+\beta_{E}}\left(\frac{\cos \left(\lambda_{n}(x-1 / 2)\right)}{\cos \left(\lambda_{n} / 2\right)}-1\right), \\
\theta_{1} & =\frac{\beta_{E} \theta_{E} V_{1} \lambda_{n}}{\left(\omega_{(\lambda n)}+1\right)\left(\alpha_{E}+\beta_{E}\right)} \frac{\sin \left(\lambda_{n}(x-1 / 2)\right)}{\cos \left(\lambda_{n} / 2\right)} \\
\sigma_{1} & =-\frac{\beta_{E} V_{1} \lambda_{n}}{\left(D \omega_{(\lambda n)}+1\right)\left(\alpha_{E}+\beta_{E}\right)} \frac{\sin \left(\lambda_{n}(x-1 / 2)\right)}{\cos \left(\lambda_{n} / 2\right)},
\end{aligned}
$$

where $\omega_{\lambda n}=\Omega_{+}\left(\lambda_{n}\right)$ and $\lambda_{n}$ is given by

$$
\frac{\alpha_{E}}{\beta_{E}}+\frac{2}{\lambda_{n}} \tan \left(\frac{\lambda_{n}}{2}\right)=0
$$

such that $(2 n-1) \pi<\lambda_{n}<2 \pi n$. This demonstrates that small perturbations to the stationary strip that break the symmetry, about $x=1 / 2$, of the network volume fraction or network stress lead to a travelling strip. This stability analysis gives insight into the polarization that a cell undergoes in order to start crawling. The model provides a connection between breaking the symmetry of the initially uniform network phase and the motion of the strip.

\subsection{Solution of the linearized initial value problem}

We consider how the stationary strip responds to a general small perturbation of its network volume fraction and network stress. We confine ourselves to the parameter regime where the linearized problem is well-posed $\left(D<1, \Psi_{E}>-1 / D\right)$ and seek a solution to the linearized initial value problem by writing arbitrary initial conditions for $\theta_{1}$ and $\sigma_{1}$ as sums over the modes as follows

$$
\begin{aligned}
& \theta_{1}=\frac{a_{0}(t)}{2}+\sum_{n \text { even }} a_{n}(t) \cos (n \pi x)+\sum_{n} b_{n}(t) \frac{\sin \left(\lambda_{n}(x-1 / 2)\right)}{\cos \left(\lambda_{n} / 2\right)}, \\
& \sigma_{1}=\frac{c_{0}(t)}{2}+\sum_{n \text { even }} c_{n}(t) \cos (n \pi x)+\sum_{n} d_{n}(t) \frac{\sin \left(\lambda_{n}(x-1 / 2)\right)}{\cos \left(\lambda_{n} / 2\right)}
\end{aligned}
$$

where $\lambda_{n}$ is given by (56). The initial conditions for $a_{n}, b_{n}, c_{n}$ and $d_{n}$ are given by the initial conditions for $\theta$ and $\sigma$, where we have expanded $\theta^{*} \sim \theta_{E}+\varepsilon \theta_{1}^{*}+\ldots$ and 
$\sigma^{*} \sim \varepsilon \sigma_{1}^{*}+\ldots$ so that

$$
\begin{aligned}
& a_{n}(0)=2 \int_{0}^{1} \theta_{1}^{*}(\zeta) \cos (n \pi \zeta) \mathrm{d} \zeta, \\
& b_{n}(0)=\frac{2 \lambda_{n} \cos \left(\lambda_{n} / 2\right)}{\lambda_{n}-\sin \left(\lambda_{n}\right)} \int_{0}^{1} \theta_{1}^{*}(\zeta) \sin \left(\lambda_{n}(\zeta-1 / 2)\right) \mathrm{d} \zeta, \\
& c_{n}(0)=2 \int_{0}^{1} \sigma_{1}^{*}(\zeta) \cos (n \pi \zeta) \mathrm{d} \zeta, \\
& d_{n}(0)=\frac{2 \lambda_{n} \cos \left(\lambda_{n} / 2\right)}{\lambda_{n}-\sin \left(\lambda_{n}\right)} \int_{0}^{1} \sigma_{1}^{*}(\zeta) \sin \left(\lambda_{n}(\zeta-1 / 2)\right) \mathrm{d} \zeta .
\end{aligned}
$$

The modes used in (57) and (58) are orthogonal on $0 \leqslant x \leqslant 1$, so substituting (57) into (43) we collect like terms to obtain

$$
D \ddot{a}_{n}+\left(\frac{D \Psi_{E}^{\prime} \theta_{E} n^{2} \pi^{2}}{\alpha_{E}+\beta_{E}}+D+1\right) \dot{a}_{n}+\left(\frac{\Psi_{E}^{\prime} \theta_{E} n^{2} \pi^{2}}{\alpha_{E}+\beta_{E}}+1\right) a_{n}=0
$$

for $n>0$ and even, along with

$$
D \ddot{b}_{n}+\left(\frac{D \Psi_{E}^{\prime} \theta_{E} \lambda_{n}^{2}}{\alpha_{E}+\beta_{E}}+D+1\right) \dot{b}_{n}+\left(\frac{\Psi_{E}^{\prime} \theta_{E} \lambda_{n}^{2}}{\alpha_{E}+\beta_{E}}+1\right) b_{n}=0,
$$

for $n>0$ an integer and

$$
D \ddot{a}_{0}+(D+1) \dot{a}_{0}+a_{0}=0 .
$$

For $D \neq 0$ these second-order, linear differential equations for $a_{n}$ and $b_{n}$ can be written as

$$
\ddot{a}_{n}+b(n \pi) \dot{a}_{n}+c(n \pi) a_{n}=0, \quad \ddot{b}_{n}+b\left(\lambda_{n}\right) \dot{b}_{n}+c\left(\lambda_{n}\right) b_{n}=0,
$$

where $b$ and $c$ are the coefficients for the growth rate quadratic and are defined in (45). Noting that we have only one initial condition for each $a_{n}$ and $b_{n}$, we differentiate (41) once with respect to $x$ and combine it with (42) to obtain a relationship between $\theta_{1}$ and $\sigma_{1}$. We then substitute in (57) and (58) and appeal to orthogonality to equate like terms to find

$$
\theta_{E}^{2} n^{2} \pi^{2} c_{n}=\left(\alpha_{E}+\beta_{E}\right) \dot{a}_{n}+\left(\alpha_{E}+\beta_{E}+\theta_{E} \Psi_{E}^{\prime} n^{2} \pi^{2}\right) a_{n}
$$

for $n>0$ and even and

$$
\theta_{E}^{2} \lambda_{n}^{2} d_{n}=\left(\alpha_{E}+\beta_{E}\right) \dot{b}_{n}+\left(\alpha_{E}+\beta_{E}+\theta_{E} \Psi_{E}^{\prime} \lambda_{n}^{2}\right) b_{n}
$$

for $n>0$ an integer and

$$
\dot{a}_{0}=-a_{0} .
$$

The first two provide initial conditions for $\dot{a}_{n}(0)$ and $\dot{b}_{n}(0)$ in terms of the known $a_{n}(0)$, $b_{n}(0), c_{n}(0)$ and $d_{n}(0)$. The third equation can be solved immediately to give

$$
a_{0}(t)=a_{0}(0) e^{-t}
$$


which we note is compatible with (65). Having found sufficient initial conditions we solve for $a_{n}$ and $b_{n}$ as follows

$$
\begin{aligned}
& a_{n}(t)=\left(\frac{\dot{a}_{n}(0)-a_{n}(0) \Omega_{-}(n \pi)}{\Omega_{+}(n \pi)-\Omega_{-}(n \pi)}\right) e^{\Omega_{+}(n \pi) t}+\left(\frac{a_{n}(0) \Omega_{+}(n \pi)-\dot{a}_{n}(0)}{\Omega_{+}(n \pi)-\Omega_{-}(n \pi)}\right) e^{\Omega_{-}(n \pi) t}, \\
& b_{n}(t)=\left(\frac{\dot{b}_{n}(0)-b_{n}(0) \Omega_{-}\left(\lambda_{n}\right)}{\Omega_{+}\left(\lambda_{n}\right)-\Omega_{-}\left(\lambda_{n}\right)}\right) e^{\Omega_{+}\left(\lambda_{n}\right) t}+\left(\frac{b_{n}(0) \Omega_{+}\left(\lambda_{n}\right)-\dot{b}_{n}(0)}{\Omega_{+}\left(\lambda_{n}\right)-\Omega_{-}\left(\lambda_{n}\right)}\right) e^{\Omega_{-}\left(\lambda_{n}\right) t},
\end{aligned}
$$

where $\Omega_{ \pm}$is given by (46). These coefficients can now be reassembled in (57) to determine the response of the network volume fraction, $\theta_{1}$, to a general, small perturbation of the network in terms of the model parameters. Using (67) and (68) we find $c_{n}$ and $d_{n}$ in terms of $a_{n}$ and $b_{n}$, so we also have an expression for $\sigma_{1}$ in terms of the initial conditions and model parameters. Taking (40) and substituting for $\theta_{1}$ via (57), we can integrate once with respect to $x$ and apply the boundary conditions $u_{1}(0)=u_{1}(1)=0$ to deduce that $u_{1}$ is given by

$$
u_{1}=\sum_{n} \frac{b_{n}+\dot{b}_{n}}{\theta_{E} \lambda_{n}}\left(\frac{\cos \left(\lambda_{n}(x-1 / 2)\right)}{\cos \left(\lambda_{n} / 2\right)}-1\right)-\sum_{n \text { even }}\left(\frac{a_{n}+\dot{a}_{n}}{\theta_{E} n \pi}\right) \sin (n \pi x) .
$$

Finally we use (51) to find that

$$
V_{1}=\left(1+\frac{\alpha_{E}}{\beta_{E}}\right) \sum_{n} \frac{b_{n}+\dot{b}_{n}}{\theta_{E} \lambda_{n}} .
$$

When the parameters are chosen so that the model is well-posed and there are some unstable wavenumbers, we are in the case depicted in Figure 1(b) where the growth rate plateaus to $\Omega_{\infty}$. This means that for large $n$ all the modes grow at a comparable rate and we cannot isolate a dominant mode on exiting the linear regime. So we are unable to predict a preferred direction for the cell solely from the model parameters. The direction of motion depends on the parameters used and the combination and relative amplitude of modes contained in the asymmetric part of the initial conditions. The speed $V_{1}$ that the strip crawls at, in response to a small perturbation of the stationary strip, can be written solely in terms of the $b_{n}$ modes. The $b_{n}$ modes in turn depend on the $d_{n}$ modes via the initial condition $\dot{b}_{n}(0)$ as seen from (68). There is no dependence on the $a_{n}$ or $c_{n}$ modes. This demonstrates explicitly that any motion of the strip, due to small perturbations of the network volume fraction and network stress in the stationary strip, is a function only of the modes which are asymmetric about $x=1 / 2$. This suggests that in order to 'pick' a direction and start moving cells must polarize their actin network, either by altering the actin volume fraction or stressing the network in a way that breaks the cell's symmetry. These results are verified by comparison to numerical simulation in $§ 5.1$.

That the small Deborah number limit of the viscoelastic model is a singular limit can be seen clearly from (63) and (64). When $D=0$ the order of the differential equations is reduced and there is no need for an initial condition on the network stress. Letting $D \rightarrow 0$ we have $D<1$, remembering that the problem is ill-posed only for $\Psi_{E}^{\prime}<-1 / D$, we see that increasingly extreme values $\Psi_{E}^{\prime}$ are required for the viscoelastic formulation to be ill-posed in the small Deborah number limit, c.f. the purely viscous model which is always well-posed (Kimpton et al, 2012). 


\section{Small growth rate limit}

It is not possible to carry out a traditional weakly nonlinear analysis, because there is no dominant wavenumber to excite. However, further analytical progress can be made in an analogous way by setting the height of the growth rate plateau to be small and positive. We define $\varepsilon=\Omega_{\infty}$ where $\varepsilon \ll 1$. Rearranging (47) we see that $\varepsilon$ now parametrizes the swelling and contractile pressure so that

$$
\frac{\partial \Psi}{\partial \theta}\left(\theta_{E} ; \varepsilon\right)=-\frac{1+\varepsilon}{D \varepsilon+1} .
$$

In order to retain $k_{c}=O(1)$, (48) indicates that we must rescale $\alpha=\varepsilon \hat{\alpha}$ and $\beta=\varepsilon \hat{\beta}$, so that we are considering a weak adhesion and weak inter-phase drag limit.

The small growth rate problem reveals three important timescales over which the cell responds to a small perturbation. The easiest to anticipate is the long timescale over which the cell polarizes and the profile of the actin network, network velocity and network stress develop to be far from their equilibrium values. We can reasonably anticipate that this will take a long time, because the parameters are such that the perturbation grows slowly. Perhaps harder to predict a priori is that there will be a short-time problem, where we see the mean value of the network volume fraction tend to $\theta_{E}$, the equilibrium volume fraction. The total amount of network in the travelling strip relaxes to the equilibrium amount on the timescale for polymerization and depolymerization. There is also an even shorter timescale, where the cell rapidly redistributes its network and the network stress. The need for all three of the these timescales will be elucidated below.

\subsection{Large time problem $t=\mathrm{O}(1 / \varepsilon)$}

Since the growth rate is small we anticipate that any small perturbation will develop over a long timescale. So, we rescale $\tau=\varepsilon t=O(1)$ and substitute the asymptotic expansions $\theta \sim \theta_{E}+\varepsilon \theta_{1}+\varepsilon^{2} \theta_{2}+\ldots, \sigma \sim \varepsilon \sigma_{1}+\varepsilon^{2} \sigma_{2}+\ldots, u \sim \varepsilon u_{1}+\varepsilon^{2} u_{2} \ldots$ and $V \sim \varepsilon V_{1}+\varepsilon^{2} V_{2}+\ldots$, as $\varepsilon \rightarrow 0$, into (23)-(25) and (28). The spatially uniform steady state satisfies the governing equations at $O(1)$ and the perturbation is governed by the following equations, obtained at $O(\varepsilon)$ :

$$
\theta_{E} \frac{\partial u_{1}}{\partial x}=-\theta_{1}, \quad \frac{\partial \theta_{1}}{\partial x}+\theta_{E} \frac{\partial \sigma_{1}}{\partial x}=0, \quad \sigma_{1}=\frac{\partial u_{1}}{\partial x}, \quad V_{1}=-\left\langle u_{1}\right\rangle,
$$

which must be solved subject to the boundary conditions $u_{1}(0, \tau)=u_{1}(1, \tau)=0$ and as before $J_{E}^{\prime}=1$. We note first that integrating $(76 a, c)$ with respect to $x$ and applying the boundary conditions shows that both the network stress and the perturbation to the network volume fraction have zero mean on the long timescale,

$$
\left\langle\theta_{1}\right\rangle=0, \quad\left\langle\sigma_{1}\right\rangle=0,
$$

where, as in (29), angular brackets are used to denote the integral over the strip. Secondly we can eliminate $u_{1}$ between $(76 a)$ and $(76 c)$ to see that at all late times the perturbation to the equilibrium network volume fraction is proportional to the network stress and they take opposite signs

$$
\theta_{1}+\theta_{E} \sigma_{1}=0
$$


The problem is underdetermined at this order so we seek a solvability condition at $O\left(\varepsilon^{2}\right)$, which gives

$$
\theta_{E} \frac{\partial}{\partial x}\left[(1-D) \frac{\partial}{\partial x}\left(\frac{\partial u_{1}}{\partial \tau}+u_{1} \frac{\partial u_{1}}{\partial x}-u_{1}\right)-a_{E}\left(\frac{\partial u_{1}}{\partial x}\right)^{2}\right]=\alpha_{E} u_{1}+\beta_{E}\left(u_{1}+V_{1}\right)
$$

where we have dropped the hats on $\alpha$ and $\beta$, and introduced the notation $\Psi_{E}^{\prime \prime}=\partial^{2} \Psi / \partial \theta^{2}\left(\theta_{E}, 0\right)$, $J_{E}^{\prime \prime}=\partial^{2} J / \partial \theta^{2}\left(\theta_{E}\right)$ and

$$
a_{E}=1-3 D+\frac{1}{2}\left(J_{E}^{\prime \prime} \theta_{E}+\theta_{E} \Psi_{E}^{\prime \prime}\right)
$$

This is a nonlinear, third-order, partial differential equation for $u_{1}$ depending on one additional function of time, $V_{1}$, which is determined by $(76 d)$. It should be solved subject to the boundary conditions $u_{1}(0, \tau)=u_{1}(1, \tau)=0$ and initial conditions that can be obtained from suitable initial conditions for $\theta_{1}$ by integrating $(76 a)$. We do not pursue a solution of this equation further, since it is sufficiently complicated to require numerical simulation, at which point we may as well solve the full nonlinear problem, which we do in $\S 5$. The integral constraint $(76 d)$ allows us to write the velocity of the crawling strip at a given time as a function of the network volume fraction at that time:

$$
V_{1}(\tau)=-\left\langle u_{1}\right\rangle=\frac{1}{\theta_{E}} \int_{0}^{1} \int_{0}^{x} \theta_{1}(\zeta, \tau) \mathrm{d} \zeta \mathrm{d} x
$$

This long time analysis does not apply to arbitrary initial conditions: it is restricted to initial conditions that satisfy (77) and (78), which motivates the short time problem below.

\subsection{Small time problem $t=\mathrm{O}(1)$}

We consider $t=O(1)$ and make the substitution $\theta \sim \theta_{E}+\varepsilon \hat{\theta}_{1}+\ldots, \sigma \sim \varepsilon \hat{\sigma}_{1}+\ldots$, $u \sim \varepsilon \hat{u}_{1} \ldots$ and $V \sim \varepsilon \hat{V}_{1}+\ldots$, as $\varepsilon \rightarrow 0$, into (23)-(25) and (28). At $O(\varepsilon)$ we find

$$
\frac{\partial \hat{\theta}_{1}}{\partial t}+\theta_{E} \frac{\partial \hat{u}_{1}}{\partial x}=-\hat{\theta}_{1}, \quad \frac{\partial \hat{\theta}_{1}}{\partial x}+\theta_{E} \frac{\partial \hat{\sigma}_{1}}{\partial x}=0, \quad D \frac{\partial \hat{\sigma}_{1}}{\partial t}+\hat{\sigma}_{1}=\frac{\partial \hat{u}_{1}}{\partial x}, \quad \hat{V}_{1}=-\left\langle\hat{u}_{1}\right\rangle
$$

We can immediately integrate $(82 a, c)$ once with respect to $x$ and apply the zero Dirichlet boundary conditions for $\hat{u}_{1}$, then solve the resulting equations to find that

$$
\left\langle\hat{\theta}_{1}\right\rangle=\left\langle\hat{\theta}_{1}^{*}\right\rangle e^{-t}, \quad\left\langle\hat{\sigma}_{1}\right\rangle=\left\langle\hat{\sigma}_{1}^{*}\right\rangle e^{-t / D}
$$

where a star identifies initial conditions. We see that the mean values of the perturbation to the network volume fraction and the network stress decay to zero over this timescale and provide variables that are consistent with (77). Next we integrate (82b) twice with respect to $x$ and find that

$$
\hat{\theta}_{1}+\theta_{E} \hat{\sigma}_{1}=\left\langle\hat{\theta}_{1}\right\rangle+\theta_{E}\left\langle\hat{\sigma}_{1}\right\rangle,
$$

for all $t$. Since we know that the mean values decay this indicates that as we exit the small time limit our network volume fraction and stress variables will be consistent with 
(78). It is also possible to manipulate $(82 a-c)$ to isolate $\hat{\theta}_{1}$ and $\hat{\sigma}_{1}$ in turn and solve the resulting partial differential equations to find that

$$
\hat{\theta}_{1}=\hat{\theta}_{1}^{*}+\left\langle\hat{\theta}_{1}^{*}\right\rangle\left(e^{-t}-1\right), \quad \hat{\sigma}_{1}=\hat{\sigma}_{1}^{*}+\left\langle\hat{\sigma}_{1}^{*}\right\rangle\left(e^{-t / D}-1\right),
$$

so on this timescale the $x$ dependence of network volume fraction and stress is unaltered, both of the perturbation profiles simply translate to have zero mean. Substituting for $\hat{\theta}_{1}$ we can use $(82 a)$ and then $(82 d)$ to calculate the network velocity and the speed of the travelling strip

$$
\hat{u}_{1}=-\frac{1}{\theta_{E}}\left(\int_{0}^{x} \hat{\theta}_{1}^{*}(\zeta) \mathrm{d} \zeta-x\left\langle\hat{\theta}_{1}^{*}\right\rangle\right), \quad \hat{V}_{1}=\frac{1}{\theta_{E}}\left(\int_{0}^{1} \int_{0}^{x} \hat{\theta}_{1}^{*}(\zeta) \mathrm{d} \zeta \mathrm{d} x-\frac{1}{2}\left\langle\hat{\theta}_{1}^{*}\right\rangle\right)
$$

On this timescale the network velocity and the velocity of the travelling strip are determined by the initial conditions and independent of time. The small growth rate limit enables us to say a little more about the direction of motion on this timescale. In the case where, loosely speaking, one side of the strip has a higher than mean network volume fraction and the other side has a lower than mean network volume fraction, the side with the higher network volume fraction will become the rear of the travelling strip. We also note that $(86 b)$ is consistent with $(81)$. The short time analysis provides appropriate initial conditions for the long time problem that satisfy (77) and (78), however it will not work for arbitrary initial conditions. Initial conditions for this small time problem must satisfy (84). To resolve this limitation we consider an even shorter timescale.

\subsection{Very small time problem $t=\mathrm{O}(\varepsilon)$}

We consider a very short timescale in which there is fast flow of the network in order to modify arbitrary initial network volume fraction and stress profiles to comply with (84). We make the scalings $t=\varepsilon \tilde{t}$, where $\tilde{t}=O(1)$ and substitute $\theta \sim \theta_{E}+\varepsilon \tilde{\theta}_{1}+\ldots$, $\sigma \sim \varepsilon \tilde{\sigma}_{1}+\ldots, u \sim \tilde{u}_{1}+\ldots$ and $V \sim \tilde{V}_{1}+\ldots$, as $\varepsilon \rightarrow 0$, into (23)-(25) and (28). At leading order

$$
\begin{gathered}
\frac{\partial \tilde{\theta}_{1}}{\partial \tilde{t}}+\theta_{E} \frac{\partial \tilde{u}_{1}}{\partial x}=0, \quad D \frac{\partial \tilde{\sigma}_{1}}{\partial \tilde{t}}=\frac{\partial \tilde{u}_{1}}{\partial x}, \quad \tilde{V}_{1}=-\left\langle\tilde{u}_{1}\right\rangle, \\
\frac{\partial \tilde{\theta}_{1}}{\partial x}+\theta_{E} \frac{\partial \tilde{\sigma}_{1}}{\partial x}=\left(\alpha_{E}+\beta_{E}\right) \tilde{u}_{1}+\beta_{E} \tilde{V}_{1},
\end{gathered}
$$

First we integrate $(87 a, b)$ and apply the boundary conditions $\tilde{u}_{1}(0, \tilde{t})=\tilde{u}_{1}(1, \tilde{t})=0$ to see that

$$
\frac{\partial}{\partial \tilde{t}}\left\langle\tilde{\theta}_{1}\right\rangle=0, \quad \frac{\partial}{\partial \tilde{t}}\left\langle\tilde{\sigma}_{1}\right\rangle=0
$$

so the average network volume fraction and network stress is fixed on this timescale, but may be redistributed within the strip. We eliminate $\tilde{\sigma}_{1}, \tilde{\theta}_{1}$ and $\tilde{V}_{1}$ between (87) and (88) and rearrange to find

$$
\frac{\partial \tilde{u}_{1}}{\partial \tilde{t}}=A \frac{\partial^{2} \tilde{u}_{1}}{\partial x^{2}}+B \int_{0}^{1} \frac{\partial \tilde{u}_{1}}{\partial \tilde{t}} \mathrm{~d} x
$$

where

$$
A=\frac{\theta_{E}(1-D)}{D\left(\alpha_{E}+\beta_{E}\right)}, \quad B=\frac{\beta_{E}}{\alpha_{E}+\beta_{E}}
$$


and we note that both these constants are positive for parameter values where the growth rate plateaus.

Below we shall show that $\tilde{u}_{1} \rightarrow 0$ as $\tilde{t} \rightarrow \infty$, which implies that $\tilde{V}_{1} \rightarrow 0$ and so the right hand side of (88) vanishes for large $\tilde{t}$. Therefore, arbitrary initial conditions for $\theta$ and $\sigma$ relax on this very short timescale to profiles that satisfy (84). We note that $\tilde{u}_{1}$ and $\tilde{V}_{1}$ tending to zero is consistent with the small time analysis where the leading order terms in the network and travelling strip velocities are $O(\varepsilon)$. In order to show that $\tilde{u}_{1} \rightarrow 0$ as $\tilde{t} \rightarrow \infty$ we multiply (90) by $\tilde{u}_{1}$, write the second spatial derivative term as a divergence minus a square, integrate with respect to $x$ from 0 to 1 , apply the boundary conditions and manipulate to obtain

$$
\frac{1}{2} \frac{\mathrm{d}}{\mathrm{d} \tilde{t}}\left[\int_{0}^{1} w^{2} \mathrm{~d} x+(1-B)\left(\int_{0}^{1} \tilde{u}_{1} \mathrm{~d} x\right)^{2}\right]=-A \int_{0}^{1}\left(\frac{\partial \tilde{u}_{1}}{\partial x}\right)^{2} \mathrm{~d} x
$$

where

$$
w=\tilde{u}_{1}-\int_{0}^{1} \tilde{u}_{1} \mathrm{~d} x
$$

and we note that $1-B$ is strictly positive. The terms inside the square brackets in (92) are greater than or equal to zero. Assuming continuity, we see that as $\tilde{t} \rightarrow \infty, \partial u / \partial x$ tends to zero throughout the strip. Therefore $\tilde{u}_{1}$ tends to a constant, which is selected as zero by applying the boundary conditions.

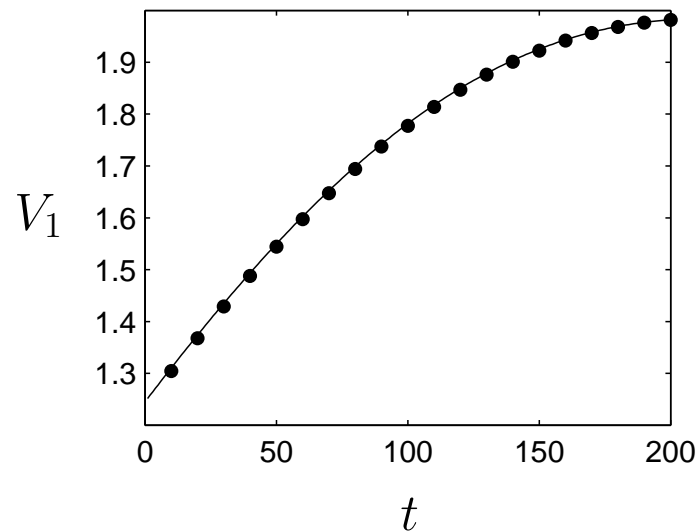

a)

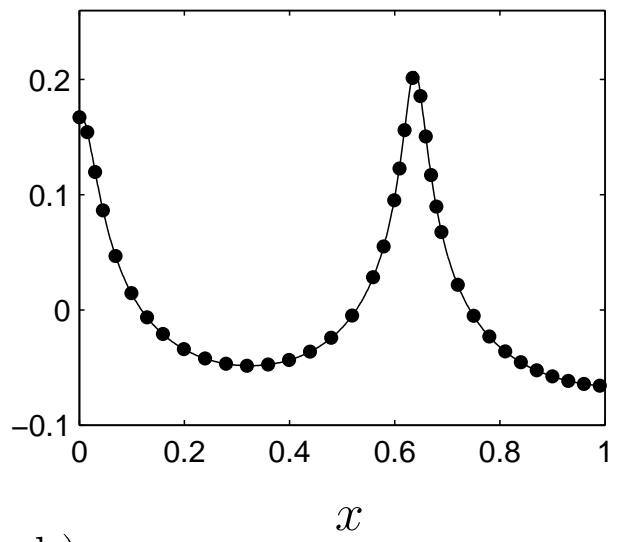

b)

Figure 3: Numerical solution to the initial value problem for initial conditions $\theta^{*}=$ $\theta_{E}+\varepsilon(\cos (3 \pi x)+1 / 4 \sin (\pi x))$ and $\sigma^{*}=\varepsilon \sin (2 \pi x)$ with parameters $\theta_{L}=0.01, \theta_{E}=0.02$, $\theta_{R}=0.03, \Psi^{*}=9800.98, \alpha^{*}=10^{-4}, \beta^{*}=5^{-4}$ and $D=0.08$, corresponding to $\varepsilon=10^{-4}$. In (a) we plot the long-time velocity of the travelling strip (solid line) and the righthand-side of (81) (dots). In (b) we check (78) at time $t=200$ by comparing $\theta_{1}$ (solid line) to $\theta_{E} \sigma_{1}$ (dots).

The small growth rate analysis has enabled us to say a little more about the direction of motion of the travelling strip. It has also revealed the three different timescales embedded in the model. First there is a rapid elastic response with redistribution of network, during 
which the strip can travel quickly at $O(1)$ speeds, (but only for a short time of $O(\varepsilon)$ ). Next we see depolymerization or polymerization of the network which causes the total amount of actin in the travelling strip to relax to the equilibrium value on the network turnover timescale: this is associated with a small constant velocity of the travelling strip. Finally we see the perturbation grow and travelling strip speed increase on the long timescale. Unfortunately the governing equation obtained on this long timescale (79) is too complicated to tackle analytically, so we proceed to present numerical solutions to the full nonlinear problem in the next section. The analysis is verified against numerical simulations in Figure 3 with the dual purpose of validating the code, see caption for details.

\section{$5 \quad$ Numerical simulations}

\subsection{Description and validation of the scheme}

We now proceed to solve numerically the two-phase, reactive, poroviscoelastic flow model stated in (23)-(32), to gain further insight into the effect of modelling the actin network as viscoelastic. We implement a fully coupled finite element scheme (see, for example, Eriksson (1996)). The resulting discretized system of nonlinear algebraic equations are solved using the PETSc libraries (Balay et al, 1997, 2010, 2011). The finite element basis used in space is continuous, piecewise linear and the scheme is fully implicit in time. An $\mathrm{LU}$ preconditioner is used to help solve the linear system arising at each Newton-like iteration and the solution at the previous time step provides an initial guess. We checked the convergence of the code as described in appendix B. In order to further validate the viscoelastic code we compare the numerical solution of a suitable initial value problem to the solution predicted by the linear stability analysis in $\S 3.3$. The two are in excellent agreement at early times as shown in Figure 4.

\subsection{Results}

The solution to the initial value problem of a small perturbation to the spatially uniform, stationary strip is displayed in Figure 4. We see in Figure 4(a) that the velocity increases initially before decaying to a constant in an oscillatory manor. The scale masks an initial transient in the speed of the travelling strip seen clearly in Figure 4(b). The linear prediction is able to match this non-monotonic behaviour because there are two roots to the growth rate equation (46) and at very early times we can observe the effect of the negative exponential in $b_{n}$, in (72). The simulation was run on a grid with five thousand spatial elements and a time step of $10^{-3}$. Comparing the computed velocity of the travelling strip to that obtained by running the same simulation on a grid with half the number of spatial elements and the same time step, or the same number of spatial elements and a time step of $2 \times 10^{-3}$, yielded a relative error in $V$ less than $1.1 \times 10^{-3}$.

The spatio-temporal behaviour of the network volume fraction $\theta$, the network velocity $u$ and the network stress $\sigma$ for this initial value problem is plotted in Figure 5. From these simulated variables we also calculate the nondimensional force exerted on the substrate 


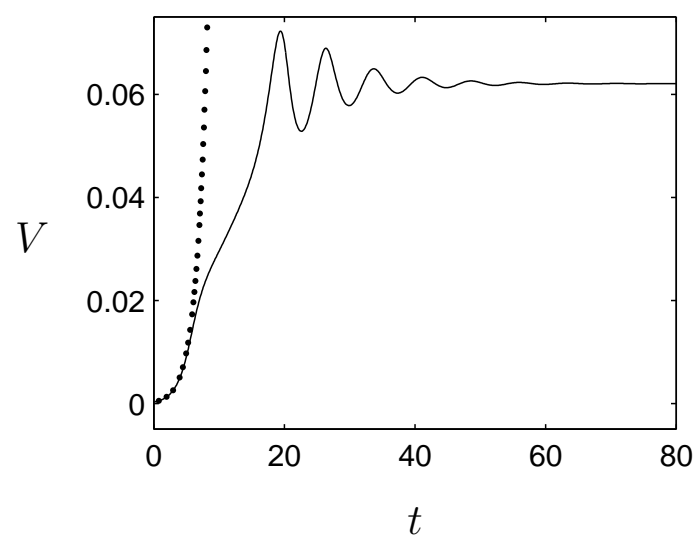

(a)

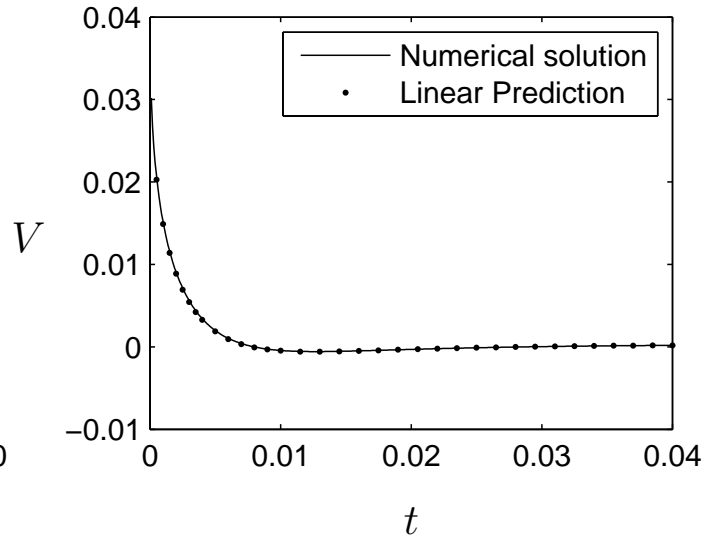

(b)

Figure 4: (a) shows the velocity of the crawling strip, $V$, as a function of time calculated numerically (solid line) and compared to the linear prediction (dots) given by (74). (b) displays a zoomed in plot of (a) showing the very early time behaviour. Parameters used are $\theta_{L}=0.01, \theta_{E}=0.02, \theta_{R}=0.03, \Psi^{*}=15000, \alpha^{*}=0.4, \beta^{*}=3$ and $D=0.1$ along with the initial conditions $\theta^{*}=\theta_{E}+\varepsilon \cos (3 \pi x)$ and $\sigma^{*}=\varepsilon \cos (\pi x)$ for $\varepsilon=10^{-4}$. Other variables of interest are plotted for the same initial value problem in Figures 5 and 6 .

by the crawling strip according to

$$
F=\beta(\theta)(u+V) .
$$

We see in Figure 5(a) that initially the volume fraction is everywhere close to the equilibrium value, then two peaks in the volume fraction develop, one at the rear of the travelling strip and one in the interior. These steepen and the interior peak travels backwards and coalesces with the peak at the rear. Subsequently smaller regions of high actin network form in the strip's interior and travel backwards, the size of these decreases with time. By $t=60$ these ripples have all but disappeared. The network velocity in Figure 5(b) is initially close to zero. Later it is negative in the bulk, displaying retrograde flow, apart from in a small region associated with the moving actin peak, where the flow is forwards. If we consider a fixed $x$ in the interior of the strip we see that after the actin peak has moved to the back of the crawling strip, the network velocity displays oscillations in time associated with the ripples seen in the network volume fraction plot. At late times the amplitude of these oscillations decays. The network stress depicted in Figure 5(c) resembles a photographic negative for the network volume fraction picture, indicating, that for these parameters at least, the network volume fraction and stress are intimately linked. Regions of high network volume fraction are associated with regions of negative network stress. Finally, the force exerted on the substrate by the crawling strip is displayed in Figure 5(d). We see that the force changes rapidly across the interior peak in the actin network. At later times the force exerted on the substrate is in the direction of motion at both ends of the travelling strip and opposing the direction of motion in the centre of the strip. There are no inertial effects in the model, the oscillations in speed of the travelling strip seem to be caused by the small local accumulations of network that travel backwards along the strip, seen as ripples in the volume fraction, velocity and stress profiles 


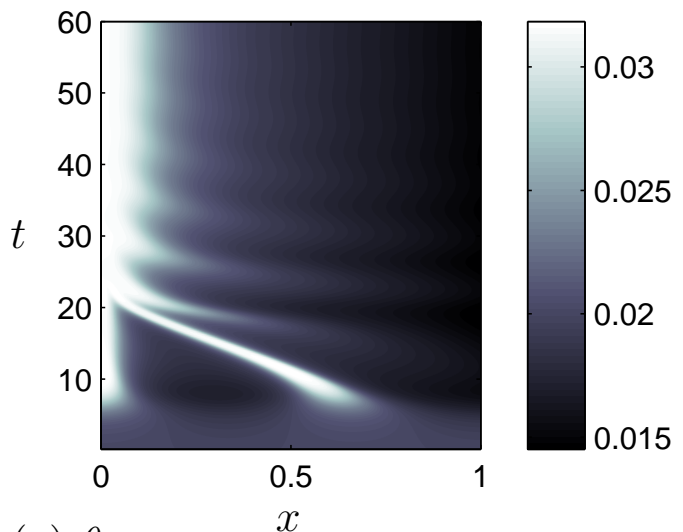

(a) $\theta$
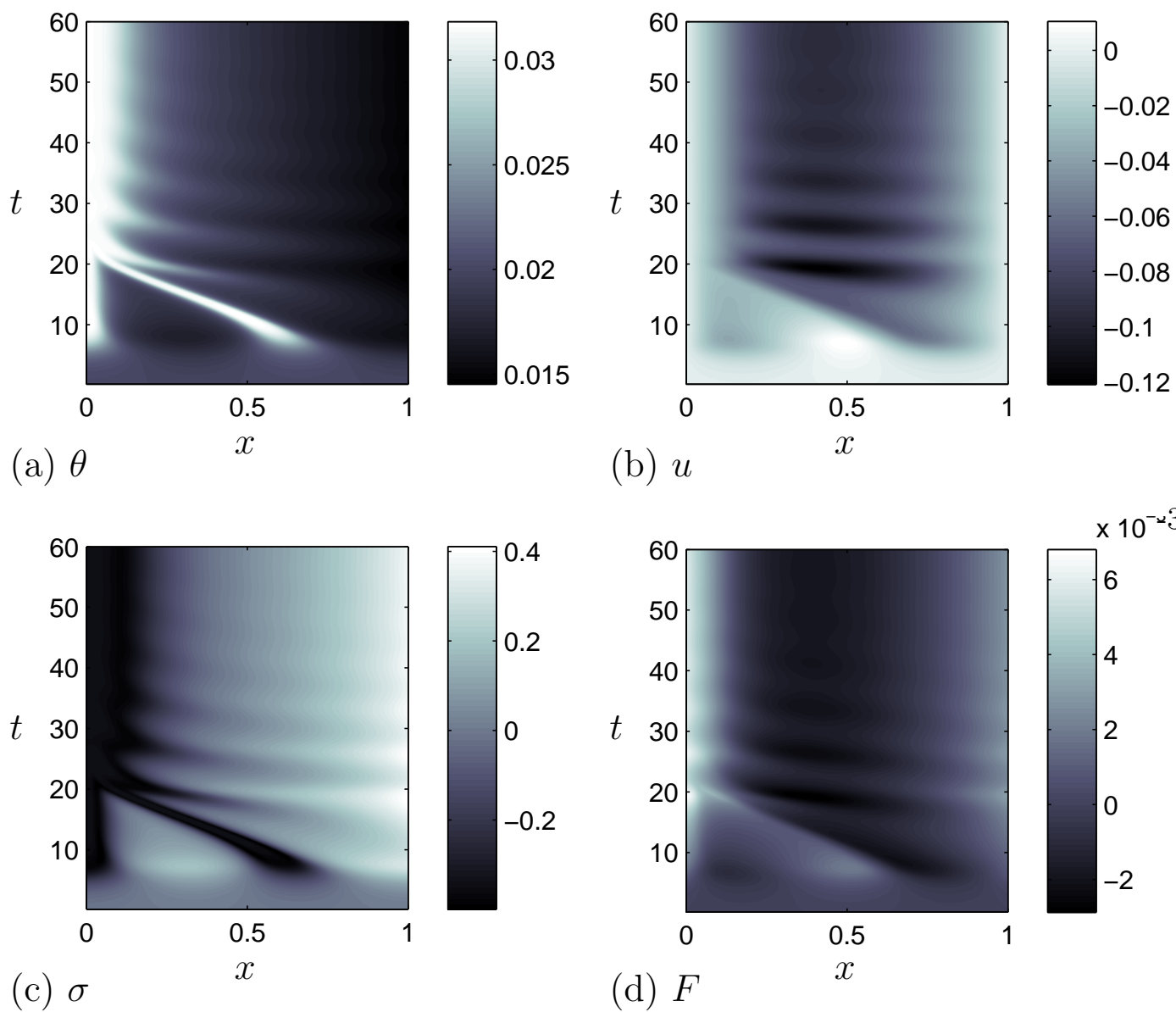

(b) $u$

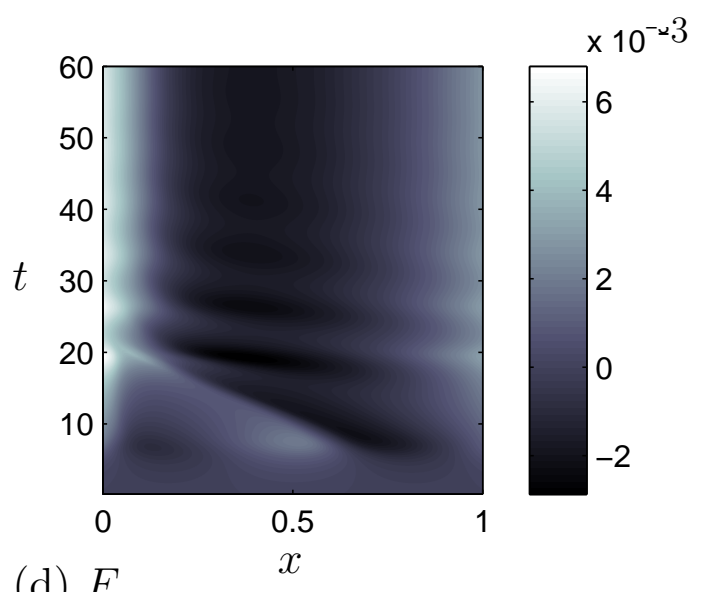

(d) $F$

Figure 5: Graphs depicting the variation in space and time of (a) the network volume fraction, (b) the network velocity, (c) the network stress and (d) the force exerted on the substrate by the crawling strip. The associated crawling strip velocity is plotted in Figure 4. Parameters and initial conditions used are stated in the caption to Figure 4.

\section{in Figure 5.}

As the speed of the travelling strip tends to a constant value we obtain a steady, travelling-wave solution as the long time limit of the unsteady problem. The maximum relative difference between the travelling-strip velocity $V$ and the average of the travelling-strip velocity for $t \in[80,100]$ is less than $1.9 \times 10^{-4}$. The steady profiles obtained are plotted in Figure 6. We see a peak in the actin network volume fraction at the rear of the travelling strip, retrograde flow of the network phase and forwards flow of the solution phase. We see that regions of high network volume fraction are associated with a negative network stress and regions of low network volume fraction are associated with a positive network stress. This is consistent with the modes found by the linear stability analysis in $(52 b, c),(54)$ and $(55)$ where the network volume fraction modes take the opposite sign to the network stress modes for positive growth rates. The network stress is not monotonic it has a local minimum in the strip interior, just in front of the peak in network volume fraction at the rear of the travelling strip. The hydrodynamic pressure is highest at the rear of the strip. The mixture stress has two interior extrema, 


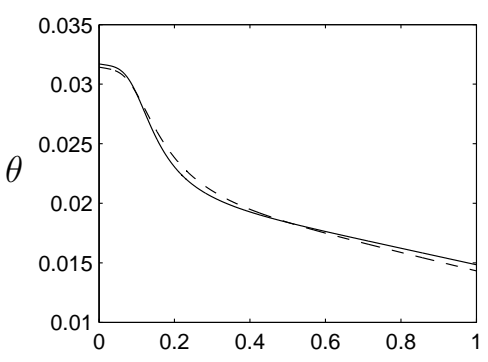

(a)

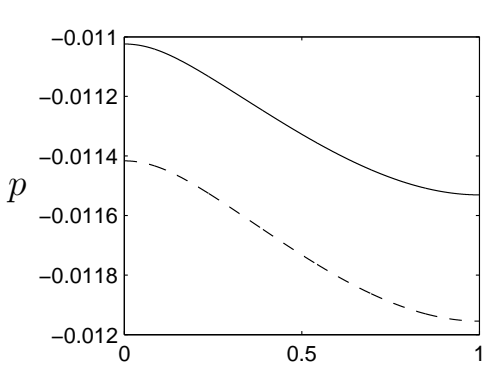

(d)

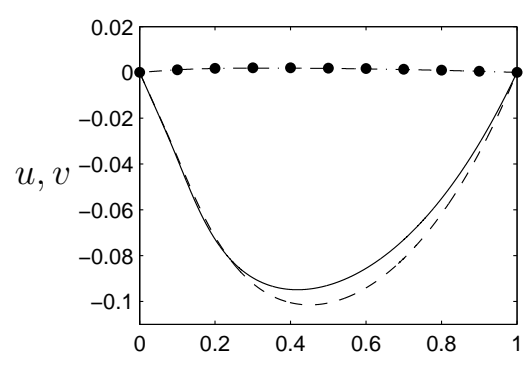

(b)

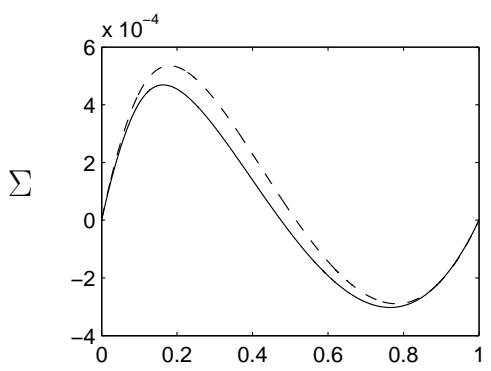

(e)


(f)

$x$

Figure 6: Steady travelling-wave profiles for the viscoelastic model (solid lines) compared to corresponding solution of the purely viscous model obtained when $D=0$ (dashed lines). (a) The network volume fraction. (b) The network velocity (solid line for $D=0.1$, dashed line for $D=0$ ) and solution velocity (dot-dashed line for $D=0.1$, dots for $D=0$ ). (c) The network stress, not calculated for $D=0$. (d) The hydrodynamic pressure. (e) The mixture stress. (f) The force exerted on the substrate by the crawling strip. The viscoelastic profiles are obtained from the initial value problem simulation at $t=200$. Parameters and initial conditions are as stated in the caption to Figure 4.

its maximum is in the back half of the travelling strip and its minimum is in the front half of the strip. The force exerted by the travelling strip on the substrate is in the direction of motion at the front and rear of the strip, but reversed for an interval in the interior. The shape of the profiles for the network volume fraction, the network and solution velocities, hydrodynamic pressure, the mixture stress and the force exerted on the substrate are qualitatively similar to the one-peak, travelling-wave, solutions found for a purely viscous model $(D=0)$ in Kimpton et al (2012), printed as dashed lines in Figure 6 for comparison. We further investigate the effect of the viscoelastic rheology on the travelling speed of the strip and the steady state profiles in the next section by varying the Deborah number $D$.

\subsection{Effect of Deborah number}

The Deborah number quantifies to what extent the network behaves as a viscous fluid or an elastic solid over our timescale of observation. As $D \rightarrow 0$ the network becomes viscous. We numerically simulate the solution to an initial value problem where the initial condition is a small perturbation to the spatially uniform steady state and plot the travelling-strip velocity in Figure 7 . We also compare the solutions to the solution of the 


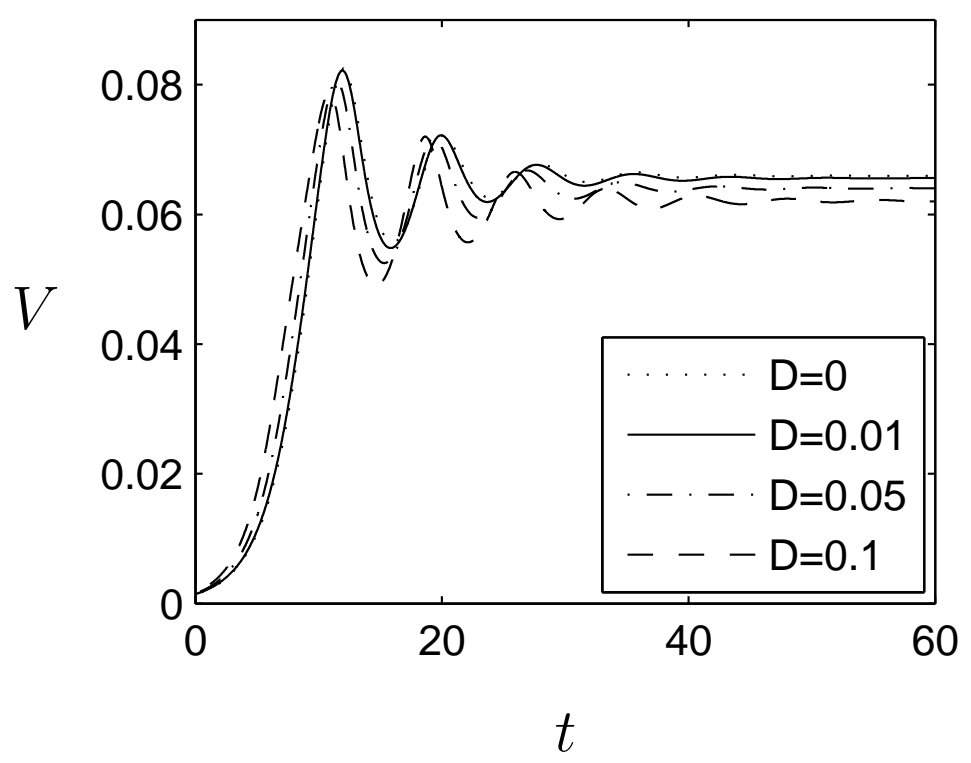

Figure 7: Velocity of the travelling strip as a function of time for different Deborah numbers. Parameters for the viscous calculation are $\theta_{L}=0.01, \theta_{E}=0.02, \theta_{R}=0.03$, $\Psi^{*}=15000, \alpha^{*}=0.4, \beta^{*}=3.0$ with the initial condition $\theta^{*}=\theta_{E}+\varepsilon \cos (\pi x)$ where $\varepsilon=10^{-4}$. For the viscoelastic simulations we have in addition $\sigma^{*}=\varepsilon \cos (\pi x)$ and $D=0.01, D=0.05$ or $D=0.1$.

equivalent problem where the network is modelled as purely viscous $(D=0)$. See caption for details of the parameters and initial conditions. We see that in all the simulations the velocity of the travelling strip initially increases, before decaying to a constant in an oscillatory manor. As the Deborah number increases the travelling-strip speed peaks sooner and slightly lower. The steady, travelling speed attained as the long time limit of the unsteady problem is slower for larger Deborah number. A small Deborah number, $D=0.01$ appears to return us almost to the purely viscous, minimal model.

Figure 8 displays the travelling-wave profiles obtained as the long time limit of the initial value problem in Figure 7 . The first observation is that an order of magnitude change in Deborah number has no qualitative impact on the travelling-wave solutions. We see in Figure 8(b) that the retrograde flow of the network near the centre of the strip is slightly slower for a larger Deborah number. A larger Deborah number also seems to result in a slightly steeper peak in the actin network volume fraction, Figure 8(a). The viscoelastic simulations in this section were run on grids with 800 or 1600 spatial elements and time steps of $1 \times 10^{-3}$ or $2 \times 10^{-3}$ and the worst relative error in crawling speed $V$ between different grids was less than $1.4 \times 10^{-3}$.

\subsection{Effect of adhesion strength}

In order to investigate the effect of adhesion strength on the speed of the travelling strip we computed steady, travelling-wave solutions by continuation in $\beta^{*}$. Figure 9 displays crawling speeds for travelling-wave solutions that all look qualitatively similar 


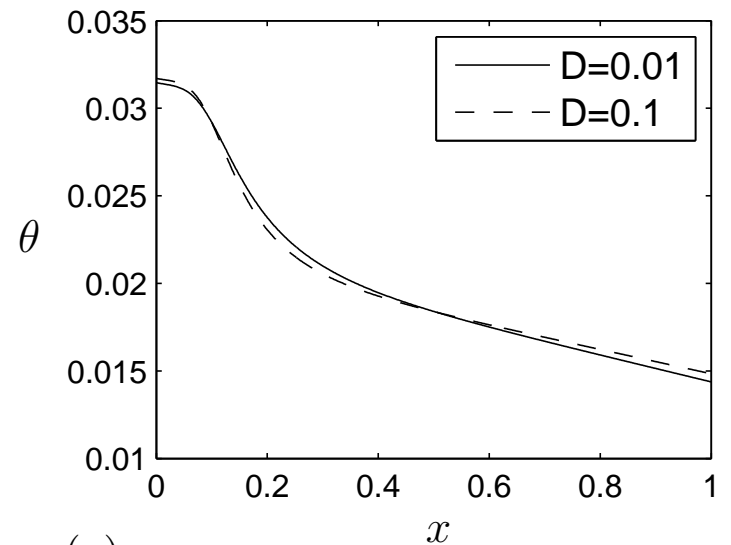

(a)

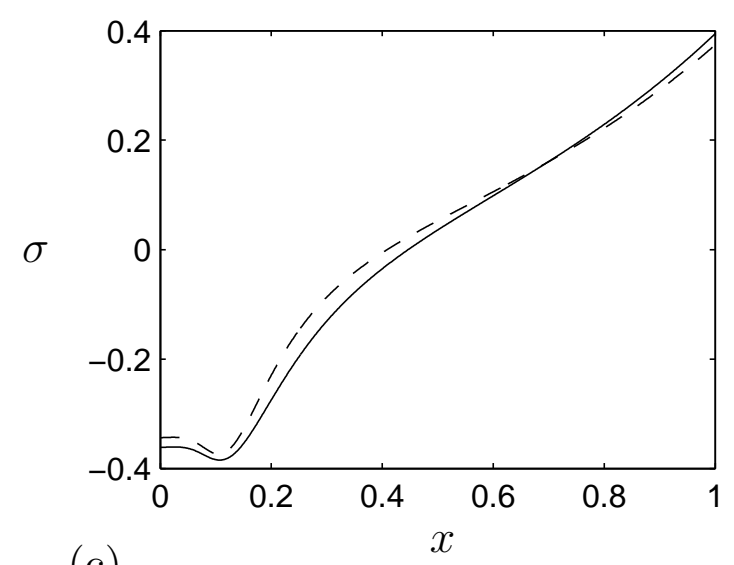

(c)

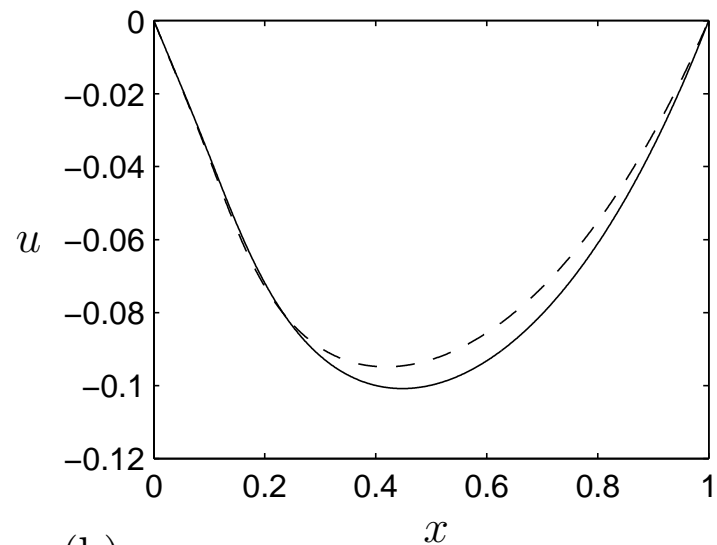

(b)

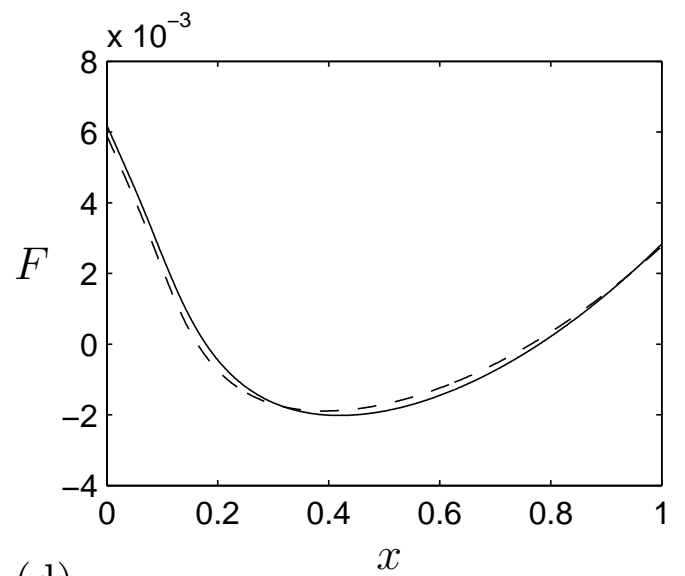

(d)

Figure 8: Comparison of the steady, travelling-wave profiles for the initial value problem in Figure 7 with $D=0.01$ (solid line) and $D=0.1$ (dashed line). (a) is the network volume fraction, (b) is the network velocity, (c) is the network stress and (d) is the force $F$ exerted on the substrate by the travelling strip, found with the unsteady code at $t=100$.

to those displayed in Figure 6. On the same graph we plot the speed of the travellingwave solutions of the equivalent purely viscous model for comparison. Both models display the biologically observed, bell-shaped dependence of travelling speed on adhesion strength. We note that for a given adhesion strength, the viscoelastic model predicts a slightly smaller crawling speed than the viscous model. The viscoelastic model also suggests a marginally smaller optimal adhesion strength. The viscoelastic solutions have all been computed on two grids, one with five thousand and one with ten thousand spatial elements. The maximum relative error in the travelling speed, $V$, computed on these grids is less than $2 \times 10^{-4}$. The maximum relative difference between $V$ and the average value of $V$ computed over the final twenty nondimensional time units is less than $3.3 \times 10^{-5}$. 


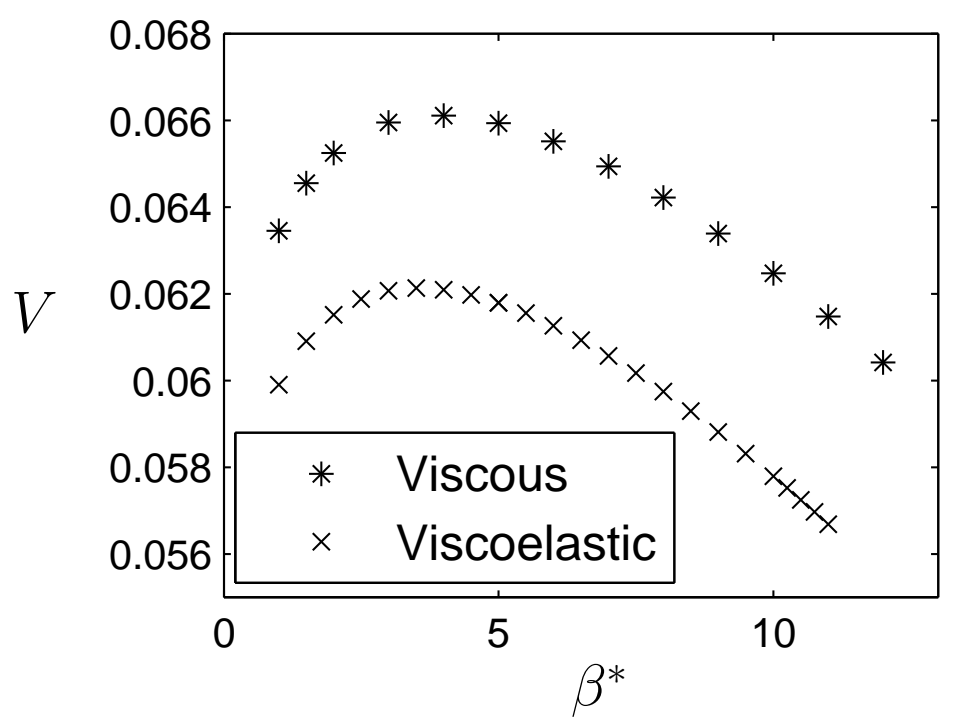

Figure 9: Travelling speed of steady travelling-wave solutions as a function of adhesion strength for both the viscoelastic model considered in this paper and the equivalent, purely viscous model. Parameters used are $\theta_{L}=0.01, \theta_{E}=0.02, \theta_{R}=0.03, \Psi^{*}=15000$, $\alpha^{*}=0.4$ and $D=0.1$.

\section{Discussion}

In this paper we formulate a one-dimensional, two-phase, reactive, poroviscoelastic model for a cell crawling on a flat substrate. The model is described in $\S 2$, we reduce the system in $\S 2.3$ and move to a travelling frame in $\S 2.5$. In order to determine which terms drive the crawling strip and to display explicitly the history dependence introduced by the viscoelastic constitutive assumption, we present a potential hodograph transformation of the governing equations in $\S 2.7$.

We also present a linear stability analysis. In $\S 3.1$ we consider perturbations to a spatially uniform steady state in order to gain insight into cell polarization. The growth rate of such a perturbation is characterized in terms of its wavenumber and the model parameters. We show that for a particular parameter regime the largest growth rate plateaus to a positive constant and the smaller root to the growth rate equation is important in understanding the early time behaviour of the linearized initial value problem presented in $\S 3.3$. In $\S 3.2$ we find the modes selected by the boundary conditions and use them to construct a solution of the linearized initial value problem in $\S 3.3$. The model displays the biologically relevant features that motion of the travelling strip requires a break in symmetry of the initial conditions, and the initial crawling velocity in the linear regime depends on both the frequency and the relative amplitude of the modes that form the initial perturbation. Another interesting feature of the viscoelastic formulation is that it yields an ill-posed system in some parameter regimes. We demonstrate in appendix C that coupling the simplest model of myosin triggered network contraction to our governing equations eliminates this problem and ensures the model is at least weakly well-posed for all parameter regimes. 
The small growth rate analysis presented in $\S 4$ reveals that two shorter timescales are required to resolve arbitrary initial conditions with the set of equations that govern the long time behaviour. On the first, very fast timescale we see rapid redistribution of the network volume fraction and stress until the perturbation to the network stress and the negative of the perturbation of the network volume fraction are proportional to each other up to some function of time. This function of time represents the average of the perturbation to the network volume fraction plus the average of the perturbation to the network stress, each multiplied by some constants. Over the next intermediate timescale this sum of averages decays, as the perturbations to the network volume fraction and the network stress translate to have zero mean, so that the profiles become proportional to the negatives of each other. During this stage the travelling strip moves at a constant velocity and the network velocity varies across the strip, but does not change with time, to leading order. If after the rapid redistribution of network (or for initial conditions where this is not necessary) we can identify one side of the cell as having a greater than mean actin volume fraction then this side will become the rear of the cell. The velocity of the network and the travelling strip then evolve on the long timescale according to (79) and (81) respectively.

In $\S 5$ we proceed to present numerical solutions of the full nonlinear governing equations. The code is first verified by checking convergence and comparison to the linear stability predictions in $\S 5.1$. We present a solution to the initial value problem, where the initial conditions are a small perturbation to the spatially uniform strip in $\S 5.2$. The numerical simulations display initial transients that decay to reveal a travelling wave solution. The travelling-wave solution displays the biologically observed high actin volume fraction at the rear of the travelling strip. It also displays the experimentally observed retrograde flow of network and reasonable profiles for the force exerted on the substrate. Our model also displays the biologically observed bell-shaped dependence of velocity on adhesion strength.

One area where the model disagrees with experimentally observed results is in its prediction of low network volume fraction at the front of the travelling strip. This suggests our constitutive assumptions have neglected at least one important feature of cell motility. A likely contender for correcting the actin volume fraction at the cell front is the inclusion of biochemical signalling, promoting polymerization of the actin network near the front of the travelling strip. We have demonstrated the suitability of the two-phase framework for modelling cell motility and used a simple model to further our understanding of it. Now additional features such as enhanced polymerization at the cell membranes can be easily incorporated without the model becoming too complex and opaque.

An interesting conclusion of this work is that the Deborah number has little qualitative effect on the solutions, as shown in $§ 5.3$. Furthermore the travelling-wave solutions are not qualitatively distinct from a family of travelling-wave solutions found for a purely viscous model in Kimpton et al (2012). This would seem to allay concerns that a purely viscous model cannot adequately capture the behaviour of a crawling cell over the relevant timescales. There is however a clear quantitative effect. In summary, we have demonstrated that viscoelasticity of the actin network is something which can be incorporated into the reactive, two-phase, flow framework and that the framework retains the biologically relevant features previously observed in a purely viscous model. These findings will be increasingly useful for future models seeking to make quantitative predictions when 
more of the parameter values are known more accurately.

\section{Acknowledgements}

This publication is based on work supported by Award No. KUK-C1-013-04, made by King Abdullah University of Science and Technology (KAUST). S.L.W. is grateful for funding from the EPSRC in the form of an Advanced Research Fellowship.

\section{A Details of the system reduction}

Potential hodograph transformations have proved useful and informative in understanding this type of system (King and Oliver, 2005; Kimpton et al, 2012). We perform a similar analysis here, seeking insight into the nature of the viscoelastic governing equations (23)(25). We introduce an integrated mass variable,

$$
s=\int_{0}^{\chi(s, t)} \theta(\xi, t) \mathrm{d} \xi, \quad \chi(0, t)=0,
$$

and transform all the dependent variables

$$
\theta(\chi(s, t), t)=\Theta(s, t), \quad u(\chi(s, t), t)=U(s, t), \quad \sigma(\chi(s, t), t)=\Lambda(s, t), \quad(96 a-c)
$$

and introduce $\phi$ and $I$ defined

$$
\phi(s, t)=\frac{1}{\Theta(s, t)}, \quad I(s, t)=-\phi(s, t) \int_{0}^{s} \phi(\eta, t) J(1 / \phi(\eta, t)) \mathrm{d} \eta
$$

to obtain, after some manipulation

$$
\begin{aligned}
\frac{\partial U}{\partial s} & =\frac{\partial \phi}{\partial t}+\frac{\partial I}{\partial s}, \\
U & =A(\phi) \frac{\partial \phi}{\partial s}+B(\phi) \frac{\partial}{\partial s}\left(\frac{\Lambda}{\phi}\right)-C(\phi) V, \\
\frac{1}{D \phi} \frac{\partial U}{\partial s} & =\frac{\partial \Lambda}{\partial t}+\frac{I}{\phi} \frac{\partial \Lambda}{\partial s}+\left(\frac{1}{D}-\frac{2}{\phi} \frac{\partial U}{\partial s}\right) \Lambda,
\end{aligned}
$$

where $A, B$ and $C$ are given by (36)-(38). It is not clear how (98)-(100) can be combined to eliminate $U$ and $\Lambda$, so we simplify the problem by considering the no polymerization/depolymerization case where $J=0$. This means that $I=0$ and $\chi$ becomes a Lagrangian variable. The transformed viscoelastic stress balance becomes

$$
\frac{\partial \Lambda}{\partial t}+E \Lambda=F
$$

where

$$
E(s, t)=\frac{1}{D}-\frac{2}{\phi} \frac{\partial \phi}{\partial t}, \quad F(s, t)=\frac{1}{D \phi} \frac{\partial \phi}{\partial t}
$$


which has the solution

$$
\Lambda=\phi^{2} e^{-t / D}\left(\frac{\Lambda^{*}}{\phi^{* 2}}+\frac{1}{D} \int_{0}^{t} \frac{e^{\xi / D}}{\phi^{3}} \frac{\partial \phi}{\partial \xi} \mathrm{d} \xi\right)
$$

where $\Lambda^{*}(x)=\Lambda(x, 0)$ is the initial condition for $\Lambda$ obtained from $\sigma^{*}$ and $\phi^{*}(s)=\phi(s, 0)$ is obtained from $\theta^{*}$, the initial condition for the network volume fraction. Now combining (98) and (99), along with $I=0$, we see that

$$
\frac{\partial \phi}{\partial t}=\frac{\partial}{\partial s}\left(A(\phi) \frac{\partial \phi}{\partial s}+B(\phi) \frac{\partial}{\partial s}\left(\frac{\Lambda}{\phi}\right)-C(\phi) V\right)
$$

Finally we can substitute for $\Lambda$ using (103) to obtain an integro-partial-differential equation for $\phi$

$$
\frac{\partial \phi}{\partial t}=\frac{\partial}{\partial s}\left(A(\phi) \frac{\partial \phi}{\partial s}+B(\phi) \frac{\partial}{\partial s}\left(\phi e^{-t / D}\left(\frac{\Lambda^{*}}{\phi^{* 2}}+\frac{1}{D} \int_{0}^{t} \frac{e^{\xi / D}}{\phi^{3}} \frac{\partial \phi}{\partial \xi} \mathrm{d} \xi\right)\right)-C(\phi) V\right),
$$

which depends on one extra unknown, $V(t)$. The interpretation of this governing equation is discussed in $\S 2.7$.

\section{B Convergence graphs}

In order to ensure that our code converges as the spatial and temporal discretization is refined we solve the initial value problem (23)-(32) on a sequence of grids. We first fix the time step at $10^{-3}$ and double the number of space elements successively from 200 to 64000 . The maximum absolute difference between the crawling velocity of the strip, $V$, calculated on successive grids is plotted against the size of the spatial element $\Delta x$ in Figure 10(a). We see that the difference decreases as the spatial grid is refined, so the code converges as the number of spatial elements increases. The spatial convergence of the numerical approximation to $V$ given by the code is order $3 / 2$. Temporal convergence is similarly demonstrated in Figure 10(b) by plotting the difference in crawling velocity of the strip as a function of step size. The temporal convergence of the numerical approximation to $V$ given by the code was shown to be first order by solving the same problem on a grid with 800 space elements for a range of time steps doubling from $1.25^{-4}$ to $4 \times 10^{-3}$. Full details of the initial conditions and parameters used are given in the caption.

\section{Myosin driven network contraction}

Some parameter choices in the viscoelastic formulation lead to an ill-posed system. Here we consider explicitly the distribution of myosin in the cell's actin network, so that the swelling contractile pressure can be a function of both local myosin concentration and local actin volume fraction. Our governing equations are given in (23)-(28) along with

$$
\frac{\partial}{\partial t}(m \theta)+\frac{\partial}{\partial x}(m \theta u)=0
$$




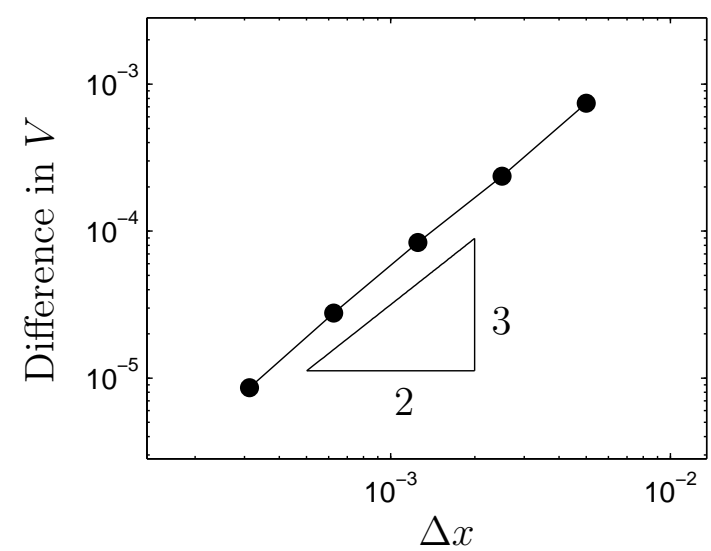

(a)

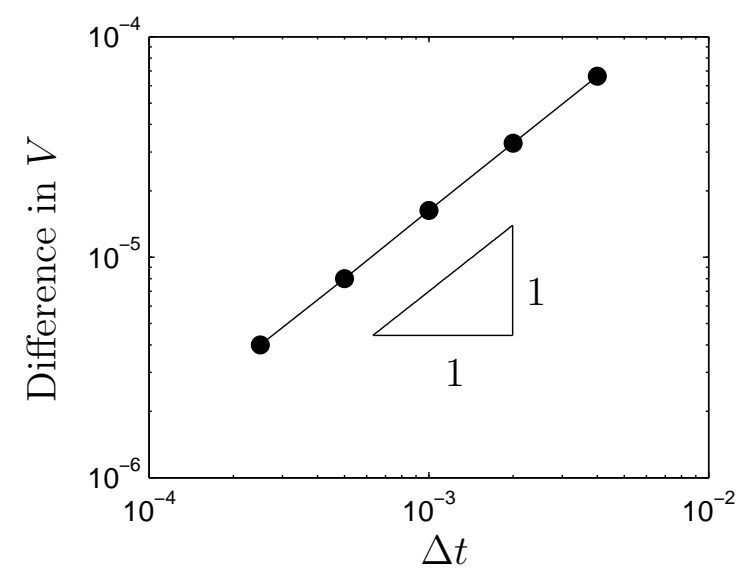

(b)

Figure 10: (a) shows the rate of spatial convergence for initial conditions $\theta^{*}=\theta_{E}+$ $10^{-4} \cos (\pi x), \sigma^{*}=10^{-4} \cos (\pi x)$ and the parameter values $\theta_{L}=0.01, \theta_{E}=0.02, \theta_{R}=$ $0.03, \Psi^{*}=15000, \alpha^{*}=0.4, \beta^{*}=3$ and $D=0.1$. (b) shows the rate of temporal convergence for the viscoelastic code with the same initial conditions and parameters as in (a) except that here $\Psi^{*}=12000$.

where $m$ is the non-dimensional concentration of bound myosin per unit volume of actin network and the above equation describes it being transported with flow of the actin network. An initial condition is required: $m(x, 0)=m^{*}(x)$, for $0<x<1$. The constitutive laws are as stated in (31a) and (32), the key difference is that modelling myosin enables us to separate the physical mechanisms for network swelling and contraction. In this section we model the network as swelling, where the swelling is reduced by myosin, so that $\Psi_{\theta}\left(\theta_{E}, M\right)>0$ and $\Psi_{m}\left(\theta_{E}, M\right)<0$, where $M$ is the spatially uniform equilibrium concentration of myosin.

In order to see whether coupling our viscoelastic model to this simple model for myosin transport eliminates the ill-posed behaviour seen in some parameter regimes for our viscoelastic model, we consider a linear stability analysis on an unbounded domain. We consider the perturbations $\theta=\theta_{E}+\varepsilon \theta_{1}+\ldots, \sigma=\varepsilon \sigma_{1}+\ldots, m=M+\varepsilon m_{1}+\ldots$, $u=\varepsilon u_{1}+\ldots$ and $V=\varepsilon V_{1}+\ldots$ and obtain at leading order

$$
\begin{aligned}
\frac{\partial \theta_{1}}{\partial t}+\theta_{E} \frac{\partial u_{1}}{\partial x} & =-\theta_{1} \\
-\Psi_{\theta E} \frac{\partial \theta_{1}}{\partial x}-\Psi_{m E} \frac{\partial m_{1}}{\partial x}+\theta_{E} \frac{\partial \sigma_{1}}{\partial x} & =\alpha_{E} u_{1}+\beta_{E}\left(u_{1}+V_{1}\right) \\
D \frac{\partial \sigma_{1}}{\partial t}+\sigma_{1} & =\frac{\partial u_{1}}{\partial x} \\
M \frac{\partial \theta_{1}}{\partial t}+\theta_{E} \frac{\partial m_{1}}{\partial t}+\theta_{E} M \frac{\partial u_{1}}{\partial x} & =0
\end{aligned}
$$

where a subscript $E$ indicates a function is evaluated at the steady state. After some 
manipulation we can eliminate the variables in favour of $\theta_{1}$, which is governed by

$$
\begin{aligned}
\left(D \frac{\partial}{\partial t}+1\right)\left(\left(\alpha_{E}\right.\right. & \left.\left.+\beta_{E}\right)\left(\frac{\partial^{2} \theta_{1}}{\partial t^{2}}+\frac{\partial \theta_{1}}{\partial t}\right)-\Psi_{\theta E} \theta_{E} \frac{\partial^{3} \theta_{1}}{\partial x^{2} t}-\Psi_{m E} M \frac{\partial^{2} \theta_{1}}{\partial x^{2}}\right) \\
& =\theta_{E} \frac{\partial^{2}}{\partial x^{2}}\left(\frac{\partial^{2} \theta_{1}}{\partial t^{2}}+\frac{\partial \theta_{1}}{\partial t}\right) .
\end{aligned}
$$

Now we consider a perturbation to the steady state, $\theta_{1} \propto \exp (\omega t+i k x)$ and obtain the following cubic equation for the growth rate of a perturbation as a function of the wavenumber of the perturbation

$$
\begin{aligned}
& D\left(\alpha_{E}+\beta_{E}\right) \omega^{3}+\left[\left(\alpha_{E}+\beta_{E}\right)(1+D)+\left(1+D \Psi_{\theta E} \theta_{E}\right) k^{2}\right] \omega^{2} \\
& \quad+\left[\alpha_{E}+\beta_{E}+\left(1+D M \Psi_{m E}+\Psi_{\theta E} \theta_{E}\right) k^{2}\right] \omega+M \Psi_{m E} k^{2}=0
\end{aligned}
$$

Seeking dominant balances in the large $k$ limit reveals that one root decays, with

$$
\omega \sim-\frac{1+D \Psi_{\theta E} \theta_{E}}{D\left(\alpha_{E}+\beta_{E}\right)} k^{2}
$$

while the two remaining roots tend to constants, $\omega_{\infty}$, satisfying

$$
\left(1+D \Psi_{\theta E} \theta_{E}\right) \omega_{\infty}^{2}+\left(1+D M \Psi_{m E}+\Psi_{\theta E} \theta_{E}\right) \omega_{\infty}+M \Psi_{m E}=0
$$

Labelling the coefficients according to $a \omega^{2}+b \omega+c=0$, we note that $a>0$ and $c<0$ so the root of the discriminant, $b^{2}-4 a c$, is bigger than $b$ in modulus and regardless of the sign on $b$ there is always one positive real root and one negative real root. Therefore, coupling a simple advection model for myosin to our viscoelastic system allows us to drive network contraction through a slightly different physical mechanism with $\Psi_{\theta E}>0, \Psi_{m E}<0$. This analysis demonstrates that on an unbounded domain the resulting system of equations will have a bounded largest growth rate that plateaus to a constant for large wavenumber perturbations, so that the governing equations are always weakly well-posed.

\section{References}

Alt W, Dembo M (1999) Cytoplasm dynamics and cell motion: two-phase flow models. Math Biosci 156(1-2):207-228

Alt W, Tranquillo R (1995) Basic morphogenetic system modeling shape changes of migrating cells: how to explain fluctuating lamellipodial dynamics. Journal of Biological Systems 3(4):905-916

Balay S, Gropp WD, Curfman McInnes L, Smith BF (1997) Modern Software Tools for Scientific Computing, Birkhäuser Press, chap Efficient Management of Parallelism in Object Oriented Numerical Software Libraries

Balay S, Brown J, Buschelman K, Eijkhout V, Gropp WD, Kaushik D, Knepley MG, Curfman McInnes L, Smith BF, Zhang H (2010) PETSc users manual. anl-95/11 revision 3.1. Argonne National Laboratory 
Balay S, Brown J, Buschelman K, Gropp WD, Kaushik D, Knepley MG, Curfman McInnes L, Smith BF, Zhang H (2011) PETSc Web page. Http://www.mcs.anl.gov/petsc.

Bausch A, Ziemann F, Boulbitch A, Jacobson K, Sackmann E (1998) Local measurements of viscoelastic parameters of adherent cell surfaces by magnetic bead microrheometry. Biophys J 75(4):2038-2049

Borm B, Requardt R, Herzog V, Kirfel G (2005) Membrane ruffles in cell migration: indicators of inefficient lamellipodia adhesion and compartments of actin filament reorganization. Exp Cell Res 302(1):83-95

Bray D (2001) Cell movements: from molecules to motility. New York, Garland Science.

Burnette D, Manley S, Sengupta P, Sougrat R, Davidson M, Kachar B, LippincottSchwartz J (2011) A role for actin arcs in the leading-edge advance of migrating cells. Nat Cell Biol 13(4):371-382

Burton K, Park J, Taylor D (1999) Keratocytes generate traction forces in two phases. Mol Biol Cell 10(11):3745-3769

Callan-Jones A, Jülicher F (2011) Hydrodynamics of active permeating gels. New J Phys 13:093,027

Callan-Jones A, Joanny J, Prost J (2008) Viscous-fingering-like instability of cell fragments. Phys Rev Lett 100(25):258,106

Cogan N, Guy R (2010) Multiphase flow models of biogels from crawling cells to bacterial biofilms. HFSP Journal 4(1):11-25

Dembo M, Harlow F (1986) Cell motion, contractile networks, and the physics of interpenetrating reactive flow. Biophys J 50(1):109-121

Dembo M, Harlow F, Alt W (1984) Cell Surface Dynamics: Concepts and Models, Marcel Deckker, chap The biophysics of cell surface motility

Doubrovinski K, Kruse K (2007) Self-organization of treadmilling filaments. Phys Rev Lett 99(22):228,104

Doubrovinski K, Kruse K (2008) Cytoskeletal waves in the absence of molecular motors. Europhys Lett 83:18,003

Doubrovinski K, Kruse K (2010) Self-organization in systems of treadmilling filaments. Eur Phys J E 31(1):95-104

Doubrovinski K, Kruse K (2011) Cell motility resulting from spontaneous polymerization waves. Phys Rev Lett 107(25):258,103

Du X, Doubrovinski K, Osterfield M (2012) Self-organized cell motility from motorfilament interactions. Biophys J 102:1738-1745 
Eriksson K (1996) Computational Differential Equations. Cambridge University Press

Giannone G, Dubin-Thaler B, Döbereiner H, Kieffer N, Bresnick A, Sheetz M (2004) Periodic lamellipodial contractions correlate with rearward actin waves. Cell 116(3):431443

Gracheva M, Othmer H (2004) A continuum model of motility in ameboid cells. Bull Math Biol 66(1):167-193

Hanein D, Horwitz A (2012) The structure of cell-matrix adhesions: the new frontier. Curr Opin Cell Biol 24:134-140

Herant M, Marganski W, Dembo M (2003) The mechanics of neutrophils: synthetic modeling of three experiments. Biophys J 84(5):3389-3413

Hinz B, Alt W, Johnen C, Herzog V, Kaiser H (1999) Quantifying lamella dynamics of cultured cells by SACED, a new computer-assisted motion analysis. Exp Cell Res 251(1):234-243

Hodge N, Papadopoulos P (2012) Continuum modeling and numerical simulation of cell motility. J Math Biol 64:1253-1279

Howell P, Kozyreff G, Ockendon J (2009) Applied solid mechanics, vol 377. Cambridge University Press

Joanny J, Jülicher F, Kruse K, Prost J (2007) Hydrodynamic theory for multi-component active polar gels. New J Phys 9:422

Jülicher F, Kruse K, Prost J, Joanny J (2007) Active behavior of the cytoskeleton. Phys Rep 449:3-28

Kimpton L, Whiteley J, Waters S, King J, Oliver J (2012) Multiple travelling-wave solutions in a minimal model for cell motility. Math Med Biol Advance Access published July 11, 2012, doi:10.1093/imammb/dqs023

King J, Oliver J (2005) Thin-film modelling of poroviscous free surface flows. Euro J Appl Math 16(04):519-553

Knapp D, Barocas V, Moon A, Yoo K, Petzold L, Tranquillo R (1997) Rheology of reconstituted type I collagen gel in confined compression. J Rheol 41(5):971-993

Kole T, Tseng Y, Jiang I, Katz J, Wirtz D (2005) Intracellular mechanics of migrating fibroblasts. Mol Biol Cell 16:328-338

Kruse K, Joanny J, Jülicher F, Prost J, Sekimoto K (2005) Generic theory of active polar gels: a paradigm for cytoskeletal dynamics. Eur Phys J E 16:5-16

Kuusela E, Alt W (2009) Continuum model of cell adhesion and migration. J Math Biol 58(1):135-161

Larripa K, Mogilner A (2006) Transport of a 1D viscoelastic actin-myosin strip of gel as a model of a crawling cell. Physica A 372(1):113-123 
Lee J, Leonard M, Oliver T, Ishihara A, Jacobson K (1994) Traction forces generated by locomoting keratocytes. J Cell Biol 127(6):1957-1964

Levayer R, Lecuit T (2011) Biomechanical regulation of contractility: spatial control and dynamics. Trends Cell Biol 22(2):61-81

Moeendarbary E, Valon L, Fritzsche M, Harris A, Moulding D, Thrasher A, Stride E, Mahadevan L, Charras G (2013) The cytoplasm of living cells behaves as a poroelastic material. Nat Mater 12(3):253-261

Mofrad M (2009) Rheology of the cytoskeleton. Annu Rev Fluid Mech 41:433-453

Mogilner A (2009) Mathematics of cell motility: have we got its number? J Math Biol 58(1):105-134

Ohsumi T, Flaherty J, Evans M, Barocas V (2008) Three-dimensional simulation of anisotropic cell-driven collagen gel compaction. Biomech Model Mechanobiol 7(1):5362

Oliver J, King J, McKinlay K, Brown P, Grant D, Scotchford C, Wood J (2005) Thinfilm theories for two-phase reactive flow models of active cell motion. Math Med Biol 22(1):53

Palecek SP, Loftus JC, Ginsberg MH, Lauffenburger DA, Horwitz AF (1997) Integrinligand binding properties govern cell migration speed through cell-substratum adhesiveness. Nature 385:537-540

Pollard T, Borisy G (2003) Cellular motility driven by assembly and disassembly of actin filaments. Cell 112(4):453-465

Ridley A (2011) Life at the leading edge. Cell 145(7):1012-1022

Rottner K, Stradal T (2011) Actin dynamics and turnover in cell motility. Curr Opin Cell Biol 23:569-578

Rubinstein B, Jacobson K, Mogilner A (2005) Multiscale two-dimensional modeling of a motile simple-shaped cell. Multiscale Model Simul 3(2):413-439

Rubinstein B, Fournier M, Jacobson K, Verkhovsky A, Mogilner A (2009) Actin-myosin viscoelastic flow in the keratocyte lamellipod. Biophys J 97(7):1853-1863

Sakamoto Y, Prudhomme S, Zaman M (2011) Viscoelastic gel-strip model for the simulation of migrating cells. Ann Biomed Eng 39(11):2735-2749

Sarvestani A, Jabbari E (2009) Analysis of cell locomotion on ligand gradient substrates. Biotechnol Bioeng 103(2):424-429

Schaub S, Bohnet S, Laurent V, Meister J, Verkhovsky A (2007) Comparative maps of motion and assembly of filamentous actin and myosin II in migrating cells. Mol Biol Cell 18(10):3723-3732 
Shao D, Levine H, Rappel W (2012) Coupling actin flow, adhesion, and morphology in a computational cell motility model. PNAS 109(18):6851-6856

Svitkina T, Verkhovsky A, McQuade K, Borisy G (1997) Analysis of the actin-myosin II system in fish epidermal keratocytes: mechanism of cell body translocation. J Cell Biol 139(2):397-415

Vallotton P, Gupton S, Waterman-Storer C, Danuser G (2004) Simultaneous mapping of filamentous actin flow and turnover in migrating cells by quantitative fluorescent speckle microscopy. PNAS 101(26):9660-9665

Vallotton P, Danuser G, Bohnet S, Meister J, Verkhovsky A (2005) Tracking retrograde flow in keratocytes: news from the front. Mol Biol Cell 16(3):1223-1231

Verkhovsky A, Svitkina T, Borisy G (1999) Self-polarization and directional motility of cytoplasm. Curr Biol 9(1):11-20

Wottawah F, Schinkinger S, Lincoln B, Ananthakrishnan R, Romeyke M, Guck J, Käs J (2005) Optical rheology of biological cells. Phys Rev Lett 94(9):98,103

Yamaoka H, Matsushita S, Shimada Y, Adachi T (2012) Multiscale modeling and mechanics of filamentous actin cytoskeleton. Biomech Model Mechanobiol 11:291-302

Zajac M, Dacanay B, Mohler W, Wolgemuth C (2008) Depolymerization-driven flow in nematode spermatozoa relates crawling speed to size and shape. Biophys J 94(10):38103823

Ziebert F, Swaminathan S, Aranson I (2012) Model for self-polarization and motility of keratocyte fragments. J R Soc Interface 9:1084-1092 



\section{RECENT REPORTS}

12/111 Exploiting the Synergy Between Carboplatin and ABT-737 in the Treatment of Ovarian Carcinomas

Jain

Richardson

Meyer-Hermann

Byrne

12/112 The integration of hormonal signaling networks and mobile mi-

Muraro croRNAs is required for vascular patterning in Arabidopsis roots

Pound

Help

Lucas

Chopard

Byrne

Godin

Hodgman

King

Pridmore

Helariutta

Bennett

Bishopp

12/113 Fast solution of Cahn-Hilliard Variational Inequalities using Im- Bosch plicit Time Discretization and Finite Elements Stoll

Benner

12/114 An Embedding Technique for the Solution of Reaction-Diffusion Equations on Algebraic Surfaces with Isolated Singularities

Rockstroh

März

Ruuth

12/115 Mathematicians at the Movies: Sherlock Holmes vs. Professor

Moulton Moriarty

Goriely

13/01 Rotation, inversion, and perversion in anisotropic elastic cylindri-

Goriely cal tubes and membranes

Tabor

13/02 Drop spreading and penetration into pre-wetted powders

Marston

Sprittles

Zhu

Li

Vakarelski

Thoroddsen

13/03 On the mechanics of thin films and growing surfaces

Holland

Kosmata

Goriely

Kuhl

Ketcheson

Macdonald

Ruuth

13/05 Simple computation of reaction-diffusion processes on point clouds

Macdonald

Merriman

Ruuth

Biddle von Glehn

Macdonald

März 
13/11 Twist and stretch of helices: All you need is Love

Đuričković

Goriely

Maddocks

13/12 Switch on, switch off: stiction in nanoelectromechanical switches

Wagner

Vella

13/13 Pinning, de-pinning and re-pinning of a slowly varying rivulet

Paterson

Wilson

Duffy

13/14 Travelling-wave similarity solutions for a steadily translating slen- Yatim der dry patch in a thin fluid film

Duffy

Wilson

13/15 A stochastic model for early placental development

Cotter

Klika

Kimpton

Collins

Heazell

13/16 Experimentally-calibrated population of models predicts and ex-

Britton plains inter-subject variability in cardiac cellular electrophysiology

Bueno-Orovio

Van Ammel

Lu

Towart

Gallacher

Rodriguez

13/17 Elastometry of deflated capsules elastic moduli from shape and wrinkle analysis

Knoche

Vella

Aumaitre

Degen

Rehage

Cicuta

Kierfeld

13/18 The effect of a concentration-dependent viscosity on particle

Herterich transport in a channel flow with porous walls

Griffiths

Field

Vella

Copies of these, and any other OCCAM reports can be obtained from:

Oxford Centre for Collaborative Applied Mathematics

Mathematical Institute

24 - 29 St Giles'

Oxford

OX1 3LB

England 
www.maths.ox.ac.uk/occam 